OPEN ACCESS

Edited by:

Wuelton Monteiro,

Fundação de Medicina Tropical Doutor Heitor Vieira Dourado

(FMT-HVD), Brazil

Reviewed by:

Jay William Fox,

University of Virginia, United States

Adriana Malheiro,

Federal University of Amazonas, Brazil

${ }^{*}$ Correspondence:

Rachael Y. M. Ryan

rachael.ryan1@jcu.edu.au

John J. Miles

john.miles@jcu.edu.au

Specialty section: This article was submitted to Vaccines and Molecular Therapeutics, a section of the journal Frontiers in Immunology

Received: 30 January 2021 Accepted: 01 April 2021 Published: 10 May 2021

Citation:

Ryan RYM, Seymour J, Loukas A, Lopez JA, Ikonomopoulou MP and

Miles JJ (2021) Immunological Responses to Envenomation.

Front. Immunol. 12:661082. doi: 10.3389/fimmu.2021.661082

\section{Immunological Responses to Envenomation}

\author{
Rachael Y. M. Ryan ${ }^{1,2,3 *}$, Jamie Seymour ${ }^{1,2}$, Alex Loukas ${ }^{1,2}$, J. Alejandro Lopez ${ }^{3,4}$, \\ Maria P. Ikonomopoulou ${ }^{5,6}$ and John J. Miles ${ }^{1,2,7 *}$ \\ 1 Division of Tropical Health and Medicine, Australian Institute of Tropical Health and Medicine, James Cook University, \\ Cairns, QLD, Australia, ${ }^{2}$ Centre for Molecular Therapeutics, Australian Institute of Tropical Health \& Medicine, James Cook \\ University, Cairns, QLD, Australia, ${ }^{3}$ School of Environment and Sciences, Griffith University, Nathan, QLD, Australia, ${ }^{4}$ QIMR \\ Berghofer Medical Research Institute, The University of Queensland, Herston, QLD, Australia, ${ }^{5}$ Translational Venomics \\ Group, Madrid Institute for Advanced Studies (IMDEA) in Food, CEI UAM+CSIC, Madrid, Spain, 6 Institute for Molecular \\ Bioscience, University of Queensland, Brisbane, QLD, Australia, ${ }^{7}$ Centre for Tropical Bioinformatics and Molecular Biology, \\ James Cook University, Cairns, QLD, Australia
}

Venoms are complex mixtures of toxic compounds delivered by bite or sting. In humans, the consequences of envenomation range from self-limiting to lethal. Critical host defence against envenomation comprises innate and adaptive immune strategies targeted towards venom detection, neutralisation, detoxification, and symptom resolution. In some instances, venoms mediate immune dysregulation that contributes to symptom severity. This review details the involvement of immune cell subtypes and mediators, particularly of the dermis, in host resistance and venom-induced immunopathology. We further discuss established venom-associated immunopathology, including allergy and systemic inflammation, and investigate Irukandji syndrome as a potential systemic inflammatory response. Finally, this review characterises venom-derived compounds as a source of immune modulating drugs for treatment of disease.

\footnotetext{
Keywords: venom, detoxification, innate immunity, adaptive immunity, immunopathology, Irukandji syndrome, venom allergy, systemic inflammation
}

\section{INTRODUCTION}

Venoms are complex mixtures of proteins, peptides, biogenic amines, and salts produced by a diverse range of animals for predation, protection, and competition (1-4). In humans, needle-like stinging apparatuses inject venom compounds into dermal-epidermal junctions, capillary vessels, and skeletal muscle fibres (5). The consequences of envenomation range from innocuous to lethal $(6,7)$.

As the initial site of venom's interaction with the immune system, the dermis provides protection through physical, chemical, and cellular defence mechanisms $(8,9)$. Prominent defenders in the dermal immune network include keratinocytes, endothelial cells, and tissue-resident and infiltrating immune cells for fast and non-specific responses (innate) and acquired long term protection (adaptive) $(8,9)$. The primary role of these cells is host defence. However, venom-mediated immune dysregulation can contribute to envenomation severity (10). Accordingly, this review discusses both the protective and pathological responses of barrier cells and the immune system towards venom compounds. 


\section{INNATE RESPONSES TO ENVENOMATION}

Defence against envenomation requires an acute response achieved by the body's innate immune system. Innate mechanisms comprise barrier and cellular defences for immediate but non-specific resistance to foreign bodies (such as venom compounds), injuries, and pathogens. Physical barriers (skin and mucosal membranes) and secretions (chemical substances and enzymes) along with resident and infiltrating immune cells provide readily available protection without requiring prior exposure to the damaging compounds (11). Instead, sentinel and scavenger cells express receptors that sense evolutionarily conserved structures common to microbes, cellular stress, and harmful substances (12).

A wide diversity of innate signalling receptor and response types is responsible for efficient detection and neutralisation/ elimination of various host threats (12). The detection of danger or stress signals initiates proinflammatory events. Broadly, these include the production of cytokines and chemokines for immune cell recruitment/activation, the release of antimicrobial peptides that directly kill pathogens, the phagocytosis and destruction of foreign particles and microbes, the generation of reactive oxygen species (ROS), reactive oxygen intermediates, and reactive nitrogen intermediates, and the release of enzymes with potent protein degrading and microbicidal properties (11).

Regulated innate effector functions are also critical for tissue repair and homeostasis (13). In addition, the presentation of foreign macromolecules, required for the establishment of acquired (adaptive) immune responses, is achieved by innate antigen-presenting cells (APC), including dendritic cells (DCs), monocytes (MNCs), and macrophages (MФ) (11). Likewise, plasma proteins, including those of the complement system (an ancient protein defensive system), promote inflammation or directly kill pathogens (14).

Detection of venom compounds by innate mechanisms initiates inflammatory reactions critical to host protection, venom detoxification, and ultimately the resolution of symptoms $(15,16)$. Participation by the epidermis, endothelium, neutrophils, MNCs, MФs, mast cells, and soluble effector mediators increases host resistance to the damaging events of bites and stings. Yet, as discussed below, many venom constituents can augment the activity of these components leading to venom-induced, immune-mediated host damage.

\section{Epidermis (Keratinocytes)}

The epidermis, the outermost layer of the skin, comprises $95 \%$ keratinocytes arranged in four layers (17). Tight junctions formed by keratinocyte-derived proteins provide a physical barrier from the external environment and structural support for Langerhans cells (epidermal-resident DCs), melanocytes, Merkel cells (tactile epithelial cells), and sensory neurons (18). Keratinocytes serve important sentinel and proinflammatory functions, where cross-talk between keratinocytes and cells of the dermal-epidermal junction direct immune cell function and maturation during both initial and late phases of inflammation (19). Like cells of the immune system, keratinocytes express cytokine receptors and pattern-recognition receptors (PRRs), enabling the detection of pathogen-, damage-, and venomassociated molecular patterns (PAMPs, DAMPs, and VAMPS) $(20,21)$. Activation of keratinocyte proinflammatory genes, such as by venom compounds, initiates the synthesis and release of cytokines, nitric oxide (NO), and alarmins, stimulating resident immune cells and attracting immune cell infiltration $(20,21)$.

To counteract this defensive barrier, many animal venoms contain matrix metalloproteinase (MMP) and L-Amino acid oxidase (LAAO) enzymes (22-25). Venom-derived MMPs and LAAOs can induce keratinocyte cell death by autophagy, apoptosis, or necrosis (22-25). Proteolytic degradation of the dermis facilitates access of venom-derived toxins to the circulation, lymphatics, and target organs for prey/predator immobilisation (22-25). In some instances, the induction of apoptosis stimulates the overexpression of endogenous MMPs, indirectly triggering tissue destruction (26). For example, brown recluse spider (Loxosceles rufescens) bites cause significant dermonecrotic effects, systemic inflammation, and potentially death in children (26). Interestingly, the molecular mechanism underpinning the initiation of cutaneous necrosis (a common reaction in loxoscelism) involves keratinocyte-derived enzymes (26). Induction of apoptosis by Loxosceles sphingomyelinase D, the main component of Loxosceles venom, stimulates the expression/activation of secreted and membrane-bound MMP2 and MMP-9 in keratinocyte cultures (26). It has been shown that the augmented expression of MMPs has a role in the necrotic skin lesions associated with $L$. rufescens envenoming (26). Hence, tetracycline has shown protective effect against venom-induced cell death by inhibiting the activation of MMP proenzyme precursors and MMP enzymatic activity (26).

\section{Endothelium}

Throughout the vascular system, endothelial cells (ECs) line the interior surface of blood and lymphatic vessels (27). Although once considered bystanders in the inflammatory process, ECs can dictate inflammatory responses under homeostatic and pathophysiological settings (28). As a primary point of contact for bloodborne pathogens and other host assaults, including toxins, ECs play an important sentinel role (29-32). Expression of numerous PRRs, including toll-like receptors (TLRs) and receptors for tumour necrosis factor (TNF) and interleukin (IL)- $1 \beta$, enables the intravascular detection of harmful compounds, the activation of proinflammatory genes, and the alteration of the microenvironment (29-32). ECs also express major histocompatibility complex (MHC) molecules, classes I and II, and costimulatory molecules, such as the CD40 ligand (CD40L) that allows intravascular antigen presentation and ECmediated activation of effector memory $\mathrm{T}$ cells $\left(\mathrm{T}_{\mathrm{EM}}\right)$ (33). However, ECs principally modulate immune function by directing leukocyte trafficking and distribution (34-36). Leukocyte tethering, rolling, and extravasation occurs in response to highly selective expression of cell adhesion molecules (CAMs), such as intercellular adhesion molecule-1 (ICAM-1) and selectins, on the apical surface of ECs. CAMs are essential for the homing and migration of immune cells towards secondary lymphoid tissue and inflammatory foci (34-36). The 
powerful influence of ECs on immune function has led to hypothesise that immune dysregulation, such as seen in systemic inflammation, might be partially mediated by the endothelium (37). As venom compounds from different species can modulate EC function (described below), this may have important implications in venom-induced systemic inflammation or allergy.

Venom from different species can induce EC perturbations, including distortions in cellular function, morphology, cytoskeletal organisation, and cell viability (38-41). Collectively these actions alter vascular permeability and blood vessel stability (38-41). Additionally, snake and spider venoms are highly proinflammatory in EC cultures, commonly provoking the secretion of IL-6, IL-8, monocyte chemoattractant protein-1 (MCP-1/CCL2), and Regulated on Activation, Normal T Cell Expressed and Secreted (RANTES/CCL5) (42). Together, these events modify the extracellular environment and leukocyte activity in local and systemic compartments, which may have important implications for the pathology of some envenomations $(41,42)$. For instance, though neutrophil depletion abrogates Loxosceles venom-induced necrotic lesions, neutrophils are not a direct target of Loxosceles venom-derived toxins (43). While neutrophils are likely the proximal cause of inflammation and tissue destruction, direct exposure to venom does not provoke this response (43). Instead, research has shown that the venom contains EC agonists that elicit dysregulated activation and cellular damage (43). Loxosceles venom strongly stimulates EC-secretion of IL-8, a potent neutrophil chemoattractant, and low-level surface expression of E-selectin (43). Researchers have noticed an unusual activation response in neutrophils to these venom-mediated proinflammatory signals (43). Specifically, neutrophils adhere to venom-stimulated ECs via selectin-mediated tethering in a time- and dose-dependent manner, yet without transmigration (43). In culture, these sequestered leukocytes rapidly increase intracellular $\mathrm{Ca}^{2+}$ levels and release primary and secondary granules containing the lytic enzymes responsible for tissue degradation (43). Accordingly, the initiation of Loxosceles necrotic lesions appears to be dependent upon toxin-mediated EC responses (43). These findings, further to work by Paixão-Cavalcante and colleagues, suggest a role for immune-targeted (in addition to toxintargeted) therapeutic strategies for envenomation (26).

\section{Mononuclear Phagocytic System}

MNCs and MФs form a crucial phagocytic component of innate immunity. Both MNCs and MФs are highly migratory, enabling tissue surveillance, antigen capture, and migration to draining lymph nodes for antigen presentation to adaptive immune cells $(44,45)$. As such, they function primarily as sentinel phagocytes and regulators of immunity (46). MNCs and MФs secrete a wide range of cytokines and chemokines that modulate immune cell function and are potent mediators of neutrophil recruitment (47).

Many animal venoms can modulate the metabolism and function of MNCs and MФs. For example, Crotalus durissus terrificus venom (CDTV) significantly inhibits the trafficking and phagocytic capacity of rat peritoneal-resident, thioglycollateelicited, and Mycobacterium bovis strain bacille Calmette Guérin (BCG)-activated MФs, without affecting cell viability at $2 \mathrm{~h}, 4$ days, or 7 days post intraperitoneal administration (48). In contrast to these immunosuppressive effects, CDTV can enhance the production of hydrogen peroxide $\left(\mathrm{H}_{2} \mathrm{O}_{2}\right)$ and $\mathrm{NO}$ from phorbol 12-myristate 13-acetate-stimulated resident, elicited, and activated MФs (48). Further, CDTV-treatment augments cellular metabolism ex vivo. Extracted peritoneal cells showed upregulated glucose and glutamine usage and increased maximal activity of hexokinase, glucose-6-phosphate dehydrogenase, citrate synthase, and phosphate-dependent glutaminase (48). These venom-mediated actions result in amplified MФs candidacidal activity and decreased phagocytosis potential (48).

Comparably, venom from the pit viper, Bothrops alternatus (BAV), stimulates increased production of superoxide anion $\left(\mathrm{O}_{2}^{-}\right)$from isolated thioglycollate-elicited MФs (49). Again, BAV-treatment showed a limited impact on $М \Phi$ viability, as evaluated by Trypan blue exclusion, and did not interfere with MФ's adhesion or detachment capacity up to $100 \mu \mathrm{g} / \mathrm{mL}$ BAV (49). Pretreatment with the protein kinase C inhibitor, staurosporine $(14 \mathrm{nM} / \mathrm{mL})$, suppressed $\mathrm{O}_{2}^{-}$production and phagocytosis, suggesting the involvement of a PKC-dependent signalling pathway (49). However, unlike CDTV, Setubal et al. observed increased MФ complement receptor (CR3)-mediated phagocytosis following incubation with BAV (49). Phagocytosis of serum-opsonised zymosan particles was significantly higher in venom-stimulated MФs compared to vehicle control (49). It was hypothesised that increased phagocytic activity and excessive release of superoxide might be involved in the local tissue destruction caused by B. alternatus snakebite (49).

Studies using human MNCs have revealed the potent proinflammatory properties of different venom compounds. For example, venom from the Androctonus crassicauda scorpion induces IL-12p40 mRNA expression and protein secretion from purified MNCs (50). However, venom exposure also produced concentration- and time-dependent cytotoxicity, as evidenced by significant LDH release in MNC cultures (50). Further examples include a C-type lectin (BjcuL) isolated from Bothrops jararacussu snake venom that induces TNF production from resting $\mathrm{CD} 14^{+}$cells without stimulating proliferation (51). Phospholipase D from Loxosceles laeta spider venom promotes MNC migration in THP-1 cell cultures and cytokine release from skin fibroblasts (52). Bothrops snake venoms provoke the release of proinflammatory mediators, prostaglandin $\mathrm{E}_{2}\left(\mathrm{PGE}_{2}\right)$,

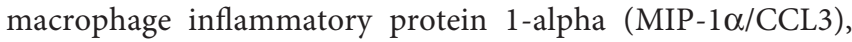
and IL-1 $\beta$, and induces activation of NF- $\kappa \mathrm{B}$ in human MNCs (53). Given the immunostimulatory role of MNCs and MФs on immune function, these data demonstrate the capacity of venom to induce systemic inflammatory responses.

Contrasting this research, Khemili et al. examined the immunosuppressive potential of ion channel modulators from scorpion venom using murine MФs (54). Voltage-gated potassium channels $\left(\mathrm{K}_{\mathrm{V}}\right)$ play a crucial role in calcium signalling and immune cell excitability (54). In the resting 
state, murine MФs predominantly express the $\mathrm{K}_{\mathrm{V}} 1.5$ subunit of the $\mathrm{K}_{\mathrm{V}} 1.5 / \mathrm{K}_{\mathrm{V}} 1.3$ heterotetrameric complex (54). Innate activation, including LPS stimulation, induces $\mathrm{K}_{\mathrm{V}} 1.3$ overexpression (54). Using non-cytotoxic concentrations of Androctonus australis hector (Aah) venom, the authors observed a voltage-independent inhibition of $\mathrm{K}_{\mathrm{V}}$ current amplitude in LPS-activated (M1) MФs (54). On the contrary, venom perfusion did not significantly decrease $\mathrm{K}_{\mathrm{V}}$ current amplitude in resting cells (54). These results suggest the presence of an ion channel blocker with a higher affinity for the $\mathrm{K}_{\mathrm{V}} 1.3$ subunit, abundant on the cell surface of activated MФs (54). However, as indicated by the authors, the downstream functional consequences of $M \Phi$ ion channel modulation requires further examination (54).

Additional immunomodulatory functions, such as TLR inhibition, have been identified using synthetic venom components (55). TLR signalling is a critical element in innate detection and $M \Phi$ activation (56). Many animal venoms contain VAMPs that strongly provoke immune stimulation via TLR engagement (56). Contrasting this, recombinant rhodostomin (Rn), a snake venom-derived disintegrin, exhibits potent TLR2 inhibition against lipopeptide-stimulated THP-1 cells (55). Incubation with $\mathrm{Rn}$ suppresses TNF, IL-1 $\beta$, and IL- 8 release and I $\mathrm{KB}$ degradation from Pam3CysSerLys4-activated cells (a TLR1/TLR2 agonist), in a dose-dependent manner (55). In THP1 cell cultures, $\mathrm{Rn}$ reverses the phosphorylation of focal adhesion kinase downstream kinases, thereby inhibiting signal transduction (55). In the caecal ligation and puncture (CLP) model of sepsis, Rn significantly suppresses CLP-induced TNF, IL-6, and MCP-1 production and reduces animal mortality (55). Histology has also revealed that Rn significantly alleviates CLPinduced tissue-damage (55). Studies such as these highlight venom as a source of compounds for drug discovery.

\section{Granulocytes (Neutrophils)}

Neutrophils are the most abundant leukocyte, constituting 40$75 \%$ of circulating white blood cells (WBC) (57). Derived from pluripotent stem cells in the bone marrow, a segmented nucleus of three to five lobes and the presence of secretory vesicles/ granules characterise mature cells (58). Although short-lived, estimates range from hours to several days, they are the first phagocyte recruited and mobilised from the bone marrow or periphery to the infection/injury $(59,60)$. Upon arrival, these granulocytes directly destroy pathogens, inactivate toxins, and mount inflammatory responses through oxidative and nonoxidative pathways $(59,60)$. Like other immune cells, neutrophils are prolific producers of cytokines and chemokines and can mount robust proinflammatory responses (61).

For non-infectious/sterile challenges, such as envenomation, exocytosis of granules/secretory vesicles releases up to 700 defensive proteins into the extracellular milieu (58). These proteins include defensins, serine proteases, neutrophil elastase, proteinase 3, cathepsin G, cytokines, and chemokines, some of which inactivate venom components through proteolytic degradation (62). An additional neutrophil defensive strategy, critical during envenomation, is the neutrophil extracellular trap or "NET". NET formation (NETosis) occurs through programmed self-destruction, whereby the release of nuclear DNA forms a sticky "net" of extracellular fibres, containing the dissemination of toxins, bacteria, and pathogens $(63,64)$. However, whether neutrophils protect against or promote venom injury is disputed. Certainly, the participation of neutrophils in venom-associated pathologies, such as dermonecrosis, has been well documented $(26,43)$. Nevertheless, neutrophilic functions, including toxin trapping and inactivation, provide critical defence against systemic injury and death (65). Additionally, neutrophil clearance of necrotic tissue is essential for muscle regeneration following snakebite (16).

Snake venom, such as from Echis carinatus, induces NETosis and ROS generation in a time- and dose-dependent manner in animal models and cell cultures (65). While these neutrophilicdefensive actions hinder venom's systemic dissemination, dense NET accumulation can block blood vessels, resulting in localised tissue damage and impeding antivenom's efficacy (65). Unfortunately, though research shows that co-treatment with DNase 1 prevents tail injury in E. carinatus experimentally envenomed rodents, mortality is significantly higher among these mice (65). Interestingly, follow-up work by Stackowicz et al. determined that localised tissue damage is neutrophil independent (66). Despite verifying that DNase-treatment does indeed reduce tail injury at the expense of survival, the study reported similar occurrences in both neutrophil-sufficient and deficient settings (66). These data suggest that extracellular DNA from multiple dying cell types, including neutrophils, mediate capillary obstruction following envenomation.

Regardless of DNA source, toxin retention inhibits systemic injury to the detriment of the localised compartments (66). NET formation and capillary obstruction can lead to severe consequences, such as amputation, which has devastating implications for victims' lives (67). Accordingly, there is an urgent need for effective therapeutics that minimise tissue necrosis and facilitate antivenom efficacy. However, given that neutrophil participation is critical in tissue repair postenvenomation, neutrophil-targeting therapies may be counterproductive (16). Hence, further research is required.

\section{Granulocytes (Mast Cells)}

Mast cells (MCs) are long-lived, tissue-resident effector cells derived from a myeloid lineage and matured under the influence of stem cell factor and cytokines (68). MCs are positioned near entry points of mucosal, epithelial, and subendothelial connective tissue to provide innate defence and perform a wide range of physiological functions that maintain tissue homeostasis (68). MCs induce killing and assist in the clearance of parasites and pathogens. For venom/toxin defence, sequestering and neutralisation occur (69). Expression of multiple PRRs on the cell surface enables rapid detection and response to immune challenges, including venom toxins. Activation of PRRs induces de novo synthesis of cytokines, chemokines, and eicosanoids to attract and stimulate other effector cells (70). A classic feature of MCs are weaponised granules, containing preformed toxic inflammatory mediators, including enzymes (tryptase, chymase, and carboxypeptidase A3), amines (histamine and heparin), and cytokines (TNF). MC activation, mediated by immunoglobulin E (IgE)-bound 
FceRI, causes rapid degranulation potentially inducing a systemic proinflammatory response $(71,72)$.

Despite the widely recognised role of MCs in allergy and anaphylactic shock, animal models have provided evidence of MCs' protective function against envenomation $(73,74)$. As reviewed by Galli et al., functional MCs enhance the survival of mice challenged with sub-lethal doses of snake (Atractaspis engaddensis; Daboia russelii), Gila monster (Heloderma suspectum), European honey bee (Apis mellifera), and scorpion (Leiurus quinquestriatus hebraeus; Centruroides exilicauda) venoms (75). The significantly higher mortality among MCdeficient mice has been attributed to the dysregulation of serine proteases (carboxypeptidase A3 and mast cell protease 4), which degrade peptides, and heparin and histamine $(69,75-77)$. In healthy individuals, the release of heparin and histamine can neutralise the effects of venom-derived toxins $(69,71)$. Adversely, the release of these amines provokes dangerous allergic symptoms in hypersensitive individuals, particularly in response to Hymenopteran venom (the venom of bees, wasps, and ants) (70).

\section{Chemical Mediators}

The immune network is vast and highly complex. Intercellular communication across the network requires small soluble protein effectors, known as cytokines (78). Cytokines (interferons, interleukins, chemokines, and growth factors) are secreted by cells to instruct and regulate the immune system's activity for protection against injury, infection, and disease (78). Biological functions include cellular activation, proliferation, differentiation, growth, and immune regulation (78). Further, as chemoattractant proteins, chemokines exert their effects via cell recruitment, migration, and adhesion (79). Like hormones, cytokines have autocrine, paracrine, or endocrine functions for localised or systemic effects (78). Broadly, they elicit either pro or anti-inflammatory action $(80,81)$. The reality, of course, is more complicated as many cytokines exhibit pleiotropic effects that are dependent on cellular source, target receptor, and the stage of the inflammatory process $(80,81)$. Additionally, immune cells adapt to the overall profile of the cytokine milieu they encounter $(80,81)$.

The expression and release of these potent chemical mediators are tightly regulated $(82,83)$. Nevertheless, infection, cancer, injury, disease (such as autoimmunity), and medical interventions (including drugs and organ transplant) can provoke dysregulation in cytokine levels resulting in devastating pathophysiological effects. Unchecked, cytokines and other proinflammatory mediators cause severe tissue destruction, systemic pathology, multiple organ failure, and potentially death $(82,83)$. Existing literature extensively describes diverse pathophysiology induced by dysregulated inflammatory mediators. These include cytokines (IL-1 $\beta$, IL-6, TNF, IFN- $\gamma$, IL-10, IL-12, and GM-CSF) and chemokines (IL-8, MCP-1, eotaxin/CCL11, IP-10/CXCL10, MDC/CCL22, MIP-1 $\alpha$, and TARC/CCL17), as well as bradykinin, eicosanoids (prostaglandins and leukotrienes), cyclooxygenases, NO, and histamine (84). Dysregulation of these mediators is associated with inflammatory and neuropathic pain $(85,86)$, tissue destruction (87), systemic inflammation (88-90), autoimmunity, and allergic reactions (91). Unsurprisingly, the same proteins are detected in the serum of victims of envenomation, where pain and systemic injury occur $(92,93)$. Notably, similar secretion profiles are also present in experimentally envenomed animals and cell cultures (92). Additionally, venoms can have detrimental effects on platelet function and components of the complement system $(94,95)$. In particular, snake venoms can trigger critical pathologies, such as venom-induced consumption, thrombocytopenia, and hemorrhage $(94,95)$.

Cytokines and their respective receptors represent important immunotherapeutic targets for numerous conditions (96). Accordingly, it might seem plausible that targeting proinflammatory cytokines, chemokines, and small molecules (or their receptors) similarly represents novel therapeutic avenues for certain envenomations. However, research in this area is still in its infancy, and to date, studies have described both beneficial and detrimental outcomes of immunosuppression during experimental envenomation. For example, the detection of snake and bee venom toxins by NOD-like receptor family, pyrin domain-containing 3 (NLRP3) inflammasome, triggers immune cell activation, potent IL-1 $\beta$ secretion, and neutrophil influx (15). Interestingly, Palm and Medzhitov showed that although inflammasome inhibition, such as seen in caspase-1deficient mice, successfully inhibited cytokine release and leukocyte influx, it unexpectedly resulted in a higher susceptibility of the mice to the noxious effects of venoms, including mortality (15). Conversely, Zoccal et al. determined that using a hexapeptide ligand for the MФ scavenger receptor (CD36) protected mice against a lethal dose of $T$. serrulatus scorpion venom through decreased production of IL-1 $\beta$, IL-6, TNF, CCL3, and PGE2, and restrained lung inflammation (97). While reduced IL-1 $\beta$ secretion and neutrophil influx was observed in both models, together, these data demonstrate the importance of innate immunodetection in the defence against bites and stings.

\section{Adaptive Responses to Envenomation}

The immune system's adaptive arm is predominantly comprised of B cells and T cells. The primary effector function of B cells is the generation of antibodies (immunoglobulins; Ig) for humoral defence (98). T cell effector functions are produced by a range of subsets, including cytotoxic $\left(\mathrm{CD}^{+}\right) \mathrm{T}$ cells and helper $\left(\mathrm{CD} 4^{+}\right) \mathrm{T}$ cells $(\mathrm{TH})$ cytotoxic $\left(\mathrm{CD}^{+}\right)$and helper $\left(\mathrm{CD} 4^{+}\right)$lymphocytes. Cytotoxic $\mathrm{CD}^{+} \mathrm{T}$ cells protect against intracellular pathogens and suppress infectious disease and tumour growth, while CD $4^{+}$ $\mathrm{T}$ cells maintain homeostasis and shape proinflammatory and regulatory immune responses (99).

Bites, stings, and intentional venom inoculation stimulate the generation of venom protein-specific antibodies (100, 101). Antibody-mediated neutralisation effectively counteracts venom activity (102). However, a primary B cell response is slow (requiring days to weeks to become fully active), while defence against rapid venom action requires an immediate response (103). As an alternative to host antibodies, antivenom, produced in large mammals and purified for 
medical purposes, can provide passive immunity to victims of life-threatening envenomation (104-106).

The following provides a simplified overview of a primary (thymus-dependent) humoral response towards envenomation. Following bite or sting, APCs, such as DCs, MNCs and MФs, capture and process venom proteins at the site of injury, promoting maturation $(100,101)$. Matured APCs migrate to secondary lymphoid tissue to present venom antigen to naïve $\mathrm{T}_{\mathrm{H}}$ cells via membrane-bound peptide-MHC II protein complexes $(100,101)$. In lymph nodes, engagement of a $\mathrm{T}$ cell receptor (TCR) with cognate peptide-MHC molecule initiates $\mathrm{T}_{\mathrm{H}}$ activation (signal 1) (107). Critical secondary signals, required for complete $\mathrm{T}$ cell activation, are provided by APCs. APCs, especially DCs, highly express ligands (including CD80 and CD86) for $\mathrm{T}$ cell co-stimulatory molecules, such as CD28 (signal 2) (108). Next, APC-derived and circulating cytokines (as well as autocrine IL-2) induce $\mathrm{T}$ cell proliferation and differentiation (signal 3). For extracellular immune challenges, such as envenomation, $\mathrm{CD}^{+} \mathrm{T}$ cells acquire a $\mathrm{T}_{\mathrm{H}} 2$ phenotype with effector functions that include B cell activation $(100,101)$.

During a primary antibody response, $\mathrm{B}$ cells require multiple stimulatory signals. The first occurs when a B cell receptor (BCR) encounters its specific soluble or membrane-bound epitope $(100,101)$. The internalised antigen is processed and displayed on the B cell surface as a peptide-MHC complex for $\mathrm{T}_{\mathrm{H}}$ presentation $(100,101)$. TCR binding triggers upregulation of co-stimulatory ligands, such as $\mathrm{CD} 40 \mathrm{~L}$, and the production of proinflammatory cytokines, including IL-4 (107). CD40L engagement with B cell CD40 mediates the recruitment of intracellular adaptor proteins essential for propagating downstream signalling (107). Additionally, cytokines secreted by primed $\mathrm{T}_{\mathrm{H}} 2$ cells provide $\mathrm{B}$ cells with accessory stimulation for the early (proliferation and clonal expansion) and later (differentiation, antibody production, and isotype switching) stages of B cell activation (109). Proliferating B cells form germinal centres (GCs) where memory B and antibodysecreting plasma cells develop. Here, B cells also undergo somatic hypermutation and isotype switching ( $\operatorname{IgM}$ and $\operatorname{IgD}$ to $\operatorname{IgG}, \operatorname{IgE}$, or $\operatorname{IgA}$ ) to generate high-affinity antibodies for robust immune responses (110).

Yet, critical though they may be, adaptive responses can also produce severe pathology (74). For example, IgE isotype switching following venom challenge can, in a percentage of hypersensitive individuals, lead to fatal allergic reactions (discussed below) (74). In addition to allergy, dysregulation of adaptive responses and loss of self-tolerance stimulate destructive auto-reactivity (111). As such, lymphocytes ( $\mathrm{T}$ cells in particular) are a target for therapeutic modulation (111). Serendipitously, venoms can contain ligands for $\mathrm{T}$ cell ion channels and receptors, able to modulate immune function with high specificity (described below) (112-121).

\section{VENOM-INDUCED IMMUNOPATHOLOGY}

Cell-specific venom-mediated immune dysregulation is described above. The following sections discuss modes of immunopathology, including venom-induced allergic reaction and systemic inflammation.

\section{Venom Allergy}

Despite a notorious reputation for venomous snakes, spiders, and jellyfish, Australia's largest proportion of venom-related fatalities occur due to anaphylactic events (122). Reflecting a global trend, honey bee (A. mellifera) stings are a significant contributor to venom injury in Australia, representing $16.3 \%$ of anaphylactic fatalities between 1997 and 2013 (123).

Venom from stinging Hymenopterans is commonly associated with allergic reactions worldwide (123). While most sting responses are localised and self-limiting, fatality can occur due to immune-mediated respiratory and/or cardiovascular failure (124). In these incidences, systemic reactions (SR) are predominantly mediated by IgE-mechanisms; however, dosedependent IgE-independent responses are also possible (7).

Among venom-sensitised individuals, SR's develop in $0.3 \%$ to $8.9 \%$ of cases $(124,125)$. Accordingly, Hymenopteran major allergens (antigens that bind IgE in greater than $50 \%$ of venomsensitive individuals) have been well-characterised (7). For honey bee venom, these hypersensitivity-inducing proteins include phospholipase A2, hyaluronidase, acid phosphatase, and dipeptidylpeptidase (124). In vespid venom (wasp and yellow jacket), Antigen 5 and phospholipase A1 are the recognised major allergens (124).

Classic IgE-mediated allergic disease begins with a sensitisation process. Keratinocytes and resident immune cells detect damage induced by noxious substances, such as venomderived compounds, stimulating the release of alarmins, cytokines (IL-4, IL-5, and IL-13), and other proinflammatory mediators required for antibody production $(126,127)$. DCs capture and process antigen for presentation to naïve $\mathrm{T}$ cells in draining lymph nodes, triggering events eventuating in plasma cell IgE antibody production $(126,127)$. Elevated IgE is a normal physiological response following a bite or sting and is not necessarily predictive of disease $(69,75,124)$. Nevertheless, in some individuals, systemic IgE levels remain elevated longer term and can trigger SR, including anaphylactic shock, after multiple stings (7).

The symptoms of immediate (Type-1) allergic reactions occur during secondary antigen challenges. When IgE encounters its cognate antigen, crosslinking of FceR1 on MCs and basophils in mucosal and epithelial tissues provoke activation and degranulation (128-130). Preformed inflammatory mediators, including histamine and proteases, are rapidly released from granules into the extracellular environment (128-130).

Histamine is chiefly responsible for the clinical consequences of Type- 1 allergic reactions $(131,132)$. Histamine's protective functions include toxin binding and deactivation $(69,75)$. In allergic disease, histamine (acting upon $\mathrm{H}_{1}$ and $\mathrm{H}_{2}$ receptors) causes smooth muscle contraction, constriction of airways, swelling of the epiglottis, and increased vascular permeability. These events lead to dangerously low blood pressure, oedema and potentially death $(131,132)$. Further to this, proinflammatory genes, stimulated during the initial phase, induces de novo synthesis of the leukotrienes (particularly 
$\mathrm{LTB}_{4}$ ), cytokines, and chemokines responsible for the late phase (or delayed-type) symptoms $(133,134)$. These mediators are potent inducers of cell activation, migration, and the influx of lymphocytes and neutrophils $(128,134)$. The incidence and severity of biphasic anaphylaxis are highly variable, and fatalities can occur, necessitating continued patient observation following the resolution of initial symptoms $(132,133,135)$.

The acute nature of fatal anaphylactic shock means death is more likely to occur in the home ( $87 \%$ of cases) than in the hospital $(122,123)$. Adrenaline autoinjectors (AAI) are an essential first-line treatment; however, death may still occur despite prompt administration $(123,136)$. Additional therapies include $\mathrm{H}_{1}$ and $\mathrm{H}_{2}$ antihistamines to counter the pathophysiological effects mediated through these receptors and critical supportive care (137, 138). For individuals with verified IgE-mediated allergy, venom immunotherapy (VIT) may generate a lifesaving tolerance to known allergens (139).

The pathogenic role of MCs and IgE-mediated granule release is well established (74). However, it has been postulated that allergy may be a barrier function disease in which cellular damage and perturbations of the epithelium and endothelium induce excessive proinflammatory responses from the resident immune cells (140). If this hypothesis is correct, the therapeutic modulation of these cells may correct the imbalanced proinflammatory response but this hypothesis has not been investigated for venom-associated allergy.

\section{Hypersensitivity to Marine Stings}

In Australia, contact with venomous marine animals and plants accounts for $9 \%$ of venom-related hospitalisations (122). The phylum Cnidaria (classes Hydrozoa, Scyphozoa, and Cubozoa) comprises approximately 10,000 jellyfish species distributed throughout the world $(138,141)$. Of these, $\sim 1 \%$ are medically relevant (142). Jellyfish stings typically trigger local or large local responses, manifesting as pain, swelling, and erythema, but are usually not life-threatening $(143,144)$. However, severe delayed cutaneous reaction, allergy, and anaphylactic shock can occur (145-147).

It may not be surprising then that in 1902 the unexpected discovery of anaphylaxis by physiologists Charles Richet and Paul Portier involved marine venom from the Portuguese manof-war (Physalia physalis) and sea anemone (148, 149). When attempting to immunize dogs against harmful venom effects, Richet and Portier found that rather than confer protection (phylaxis), a subsequent venom challenge resulted in death. Although allergy was yet to be characterised, the experiments recognised immune involvement and the term "anaphylaxis" (against protection) was coined. This discovery later won Richet the 1913 Nobel Prize in Physiology or Medicine (148, 149).

Jellyfish envenomation is the most common marine sting type, impacting fishers, surfers, and sea bathers globally. An estimated 150 million stings occur annually, with peak incidence coinciding with blooming (or swarming) seasons during warmer months (148). A characteristic feature of the phylum Cnidaria is specialised stinging organelles, known as nematocysts. Located within cnidocytes, nematocysts are explosive capsule organelles containing coiled, barbed and threadlike tubules coated in venom (150). High-velocity capsule release, triggered by physicochemical stimuli, causes inversion of tubules into harpoon-like threads able to puncture and penetrate prey and predators $(151,152)$. Through nematocysts, jellyfish venom, containing pore-forming compounds, metalloproteases, serine proteases, and phospholipases, is injected into the victim, causing paralysis of prey and in humans dysregulation of immune function, cardiac function, respiratory function, and potentially fatal outcomes (153).

Although jellyfish venom can cause severe immediate-phase and delayed-type allergic reactions, the causative allergens are mostly unknown. Chironex yamaguchii is the box jellyfish species responsible for $78 \%$ of reported stings in Japan (144). Recently, the N-linked glycoprotein, CqTX-A (a hemolytic toxin), was identified as a major allergen from this venom (144). While the underlying mechanism has yet to be elucidated, this finding has important implications as CqTX-A shares significant sequence homology with other lethal poreforming jellyfish proteins, specifically, CfTX-1 and CfTX-2 (Chironex fleckeri), CrTX-A (Carybdea rastoni), CaTX-A (Carybdea alata) and CqTX-A (Chiropsalmus quadrigatus Haeckel) (154-157).

For Hydrozoa (which includes Portuguese man-of-war), Scyphozoa (true jellyfish), and Cubozoa (box jellyfish), nematocysts are located on the tentacles, oral arms, and in some instances, the bell of the jellyfish $(158,159)$. Contact with tentacles can result in inoculation from potentially millions of nematocysts (160). Application of acetic acid and careful removal of tentacles from the victim's skin prevents the further discharge of unfired nematocysts but does not deactivate the already injected toxins (161). Once stung, venom distribution occurs via capillaries and the lymphatic system to target organs, while the barbed tubules remain embedded in the skin until clearance $(149,160)$. Tubules are allergenic scaffolds comprising carbohydrates, proteins, chitin, and mini-collagen (148, 149, 162-164). As such, it has been suggested that impaired clearance, especially of chitin, may contribute to more severe outcomes of envenomation and hypersensitivity (149).

Beyond stings, there are recently described cases of severe allergic reactions to edible jellyfish consumption (147). It has also been reported that jellyfish stings, particularly among surfers, lead to sensitisation of foods containing gamma-glutamic acid, such as fermented soybean $(165,166)$. Collectively, these data highlight the antigenic properties within jellyfish nematocysts and tissue, in addition to their venom-derived destructive potential.

\section{Systemic Inflammation}

Systemic inflammation, including cytokine release syndrome (CRS), is a life-threatening immune condition triggered in response to endotoxemia, severe viral infections (including influenza), and immunotherapies (167-169). Clinical manifestations can include fever, nausea, tachycardia, dyspnea, headache, muscle and joint pain, and in severe cases, neurotoxicity, pulmonary oedema, respiratory failure, and death (167). Certain envenomations can similarly provoke a systemic inflammatory response, most notably is scorpionism (10). 
Scorpion envenomation is another leading cause of venomassociated morbidity and mortality, affecting more than one million individuals per annum (6). Although most stings produce only local symptoms ( $81 \%$ of cases), envenoming by dangerous species can initiate a surge of endogenous neurotransmitters, adrenaline and noradrenaline, resulting in an autonomic storm and severe systemic effects $(6,10)$. Additionally, venom-derived toxins induce spontaneous acetylcholine (Ach) release from peripheral nerves, responsible for the life-threatening cardiac dysfunction seen in severe cases (170). Interestingly, these mediators are also implicated in the box jellyfish pathology, Irukandji syndrome (171).

Along with intense acute pain and distress, severe scorpion envenoming (grade III stings) produces complex pathophysiology in victims (172). Like CRS, symptoms can include respiratory distress, cardiac dysfunction, pulmonary oedema, multiple organ failure, and potentially death, especially among children and the elderly (173). These clinical consequences are principally mediated by neurotoxic peptides, able to cause hyperexcitability of the autonomic nervous system through $\mathrm{Na}^{+}, \mathrm{K}^{+}, \mathrm{Ca}^{+}$or $\mathrm{Cl}^{-}$ion channel modulation (172). Ion channel modulation is also implicated in the development of pulmonary oedema, a symptom present in many fatal sting cases (174). Research led by Comellas et al. observed decreased lung fluid clearance in Tityus serrulatus envenomed rats, postulating venom-induced impairment of $\mathrm{Na}^{+} / K_{-}^{+}$ATPase in alveolar epithelial cells as the mechanism (174).

Beyond neurotoxic effect, the immune network plays a role in significant scorpion envenomations (175). Immune participation in SR is multifactorial, involving direct antigenic activation and indirect stimulation via the neuroendocrine-immune axis (176). In addition to neurotransmitters, stings induce a rapid release of proinflammatory mediators. Elevated IFN- $\gamma$, IL-1 $\beta$, IL-6, IL-8, IL-10, and TNF have been detected in the plasma of sting patients and animal models of scorpionism (10). Scorpion venom also provokes hypersensitivity mediators, particularly histamine (177). Blockade of the histamine $\mathrm{H} 1$ receptor has shown to be protective in Androctonus australis hector envenomed mice (177). Specifically, pretreatment with hydroxyzine (H1 receptor antagonist) reduced immune cell infiltrate and oedema in the brain and spinal cord and diminished levels of circulating proinflammatory cytokines (177). Further, scorpion toxins activate components of the complement system, including the generation of anaphylatoxins, which are potent chemotactic proteins (10). As further evidence of immunological involvement, heightened dermal reactions are reported in individuals predisposed to scorpion venom, such as seen in delayed-type hypersensitivity reactions (10).

For direct immunological activation, the most extensively studied species is the Brazilian scorpion, T. serrulatus (10). Whole T. serrulatus venom (TsV) and select purified toxins are potent stimulators of innate immune cells, including MФs (56). In vitro assays have revealed that surface receptors TLR2, TLR4, CD14, and CD36 recognise TsV compounds, triggering cellular activation and production of cytokines and lipid mediators (56).
Engagement of CD14 and co-receptor TLR4 promotes NF- $\mathrm{KB}$ and AP-1 signalling pathways and transcription of potent proinflammatory genes, including IL-1 $\beta$ (10). Consequently, $\mathrm{TsV}$ stimulates cytokine release from innate immune cells in a time- and dose-dependent manner, independent from cytotoxic effect (10). In addition, NF- $\mathrm{KB}$ signalling regulates cyclooxygenase-2 (COX-2) expression and the secretion of eicosanoid, $\mathrm{PGE}_{2}$ (178). $\mathrm{PGE}_{2}$ is a lipid mediator with pleiotropic roles in the initiation and resolution of inflammation, particularly inflammatory pain (179). Among its diverse biological functions, $\mathrm{PGE}_{2}$ activates IL-1 $\beta, \mathrm{MCP}-1$, and IL-6 pathways via prostaglandin EP4 receptor signalling (179). Accordingly, IL-1 $\beta$ and its receptor (IL-1R) are strongly suppressed by EP4 antagonism (179). IL-1 $\beta$ and IL-1R are potential therapeutic targets for multiple inflammatory diseases, including scorpion envenomation (180). As such, inhibition of the $\mathrm{COX}-2 / \mathrm{PGE}_{2} / \mathrm{EP} 4$ pathway has shown a cardiopulmonary protective effect in envenomed mice $(97,170)$.

The eicosanoid leukotriene $\mathrm{B} 4\left(\mathrm{LTB}_{4}\right)$ is also upregulated in cell culture and animal plasma following treatment with whole TsV or purified toxins (178). A study by Zoccal et al. demonstrated that activation of the class B scavenger receptor, CD36, directs eicosanoid metabolism towards $\mathrm{LTB}_{4}$ via a 5lipoxygenase (5-LOX)/peroxisome proliferator-activated receptor gamma (PPAR- $\gamma$ ) pathway, opposing the events of TLR and CD14 receptor signalling (97). CD14 and TLR4 appear to be critical for TsV-induced cytokine and eicosanoid secretion (180). Further work by Zoccal and colleagues showed that $\mathrm{CD} 14^{-/-}$mice fail to produce significant levels of $\mathrm{PGE}_{2}$ or IL$1 \beta$ post- $\mathrm{TsV}$ envenomation (180). In addition, $\mathrm{CD} 36^{\mathrm{obl} / \mathrm{obl}}$ mice secrete increased levels of $\mathrm{PGE}_{2}$ and IL-1 $\beta$ post-TsV envenomation but do not produce $\mathrm{LTB}_{4}$ (180). Critically, $\mathrm{LTB}_{4}$ synthesis suppresses IL-1 $\beta$ maturation and secretion and the associated animal mortality (178). CD36, therefore, represents a novel therapeutic target for severe scorpion envenomation (180).

Mouse models of $\mathrm{TsV}$ envenomation produce autonomic dysfunction that is similar to clinically observed symptoms (170). A lethal inoculation of TsV induces sweating, ocular and nasal secretions, lethargy, and convulsions in mice, preceding cardiovascular disturbances and death (170). Observed hyperglycemia and neutrophilia are also consistent with sting patients (170). The neurotransmitters adrenaline and ACh, responsible for sympathetic and parasympathetic symptoms, respectively, are elevated in peripheral blood as well as in cardiac tissue in response to TsV (170). Treatment with atropine, a muscarinic receptor antagonist, but not propranolol, prevented venom-induced cardiovascular alterations, which are a leading cause of death in severe scorpionism (170). Curiously, despite showing systemic elevation of adrenaline, the study did not investigate the effect of an alpha-adrenergic blocking agent, such as prazosin (170).

In parallel to excessive $\mathrm{ACh}$, lethal $\mathrm{TsV}$ envenomation stimulates the systemic and cardiac secretion of $\mathrm{PGE}_{2}$ and IL$1 \beta$. Reis et al. have recently proposed IL-1R as a neuro-immune link responsible for innate heart inflammation and $\mathrm{TsV}$-induced heart failure (170). Research by their group demonstrated that 
TsV co-administered with $\mathrm{PGE}_{2}$ enhanced IL-1 $\beta$ and $\mathrm{ACh}$ release from cardio fibroblasts, an effect which was blocked by an EP receptor antagonist. In contrast, IL-1R silencing repressed $\mathrm{PGE}_{2}, \mathrm{IL}-1 \beta$ and $\mathrm{ACh}$ levels and rescued mice from fatal TsV administration (170). As such, the study determined that $\mathrm{PGE}_{2}$ amplifies IL- $1 \beta$ release, which upon binding IL-1R potentiates upregulation of $\mathrm{PGE}_{2}$ and $\mathrm{PGE}_{2}$-dependent $\mathrm{ACh}$ release post TsV envenomation (170).

Existing scorpion sting management comprises specific antiserum and symptomatic treatment, such as pain and low dose anti-inflammatory medications (6). Grade III stings and stings in children younger than 15 require intensive care (6). Polymorphism within scorpion venom-derived proteins is geographically varied, impeding the manufacture of a standardised antivenom (6). Variability in toxin immunogenicity further limits the usefulness and cost-effectiveness of antivenom production (6). Accordingly, some experts challenge the use of antiserum therapy due to insufficient neutralising capacity and the additional shock risk associated with poorly purified serum $(6,181)$.

Recently, success has been reported using novel immunebased therapies in animal models of TsV envenomation. In 2019, Zoccal et al. showed that the experimental peptide, EP80317 (a CD36 ligand), protected C57BL/6 mice against a lethal dose of $\mathrm{TsV}$ (97). Indeed, the therapeutic administration of EP80317 at $0.5 \mathrm{~h}$ and $2 \mathrm{~h}$ post-envenomation provided complete protection against a lethal dose of venom. Lymphocytes and neutrophils in the bronchoalveolar lavage fluid were significantly lower in the treatment group than venom alone. Accordingly, cAMP concentrations and proinflammatory cytokines (IL-1 $\beta$, IL-6, TNF, and CCL3) were also considerably decreased (97).

Although promising, the estimated time and cost of developing a new drug and bringing it to market is $10-15$ years and hundreds of millions of dollars (182). Conversely, drug repurposing circumvents the requirement for lengthy and expensive preclinical development. A recent in vivo study from the same group found that therapeutic administration with high dose dexamethasone (DEX) (5 mg/kg) improved TsV-induced cardiac dysfunction and reduced mortality after a fatal venom dose (170). The study showed that early treatment (15 min and $1 \mathrm{~h}$ post-inoculation) strongly suppressed $\mathrm{PGE}_{2}$ and IL-1 $\beta$ release in tissues, abrogating systemic ACh and IL1Rmediated/ACh-induced cardiac dysfunction (170).

In reviewing the effects of jellyfish venom on the immune system, Tibballs et al. highlighted similarities between the clinical features of Irukandji syndrome and scorpion envenomation (149). While the mechanisms underpinning the pathology of Irukandji syndrome have remained unresolved for decades, they may likewise involve both autonomic and inflammatory pathways. If so, immune-based therapies may also prove beneficial in severe box jellyfish envenomation and warrant further investigation.

\section{Irukandji Syndrome}

While most jellyfish stings do not require medical attention, several species found in tropical waters constitute a public health threat (141). In Australia, the box jellyfish Chironex fleckeri and
Carukia barnesi are of particular medical relevance. A high $C$. fleckeri venom dose can cause rapid and fatal cardiac arrest (183). In contrast, the smaller box jellyfish, $C$. barnesi, induces an extremely painful systemic pathology known as Irukandji syndrome (IS) (184-186).

C. barnesi was the first confirmed causative agent of IS after its namesake, Dr Jack Barnes, famously subjected himself, his nine-year-old son, and a local lifeguard to intentional envenoming in 1961 (187). Yet, due in part to the elusive nature of this highly venomous jellyfish, research over the years has failed to unravel the mechanisms behind the distinctive syndromic illness $(184,188)$. C. barnesi are small and transparent, with the medusal bell measuring $\sim 20 \mathrm{~mm}$ wide (Figure 1) $(184,189)$. As with other carybdeids, C. barnesi have a single tentacle per pedalium $(184,189)$. Both bell and tentacles are covered with nematocysts, comprising distinct venom composition (190).

A retrospective case study of 128 marine sting presentations to Cairns Base Hospital revealed a wide variation of symptom severity among individuals (186). Of the 39 patients with skin scrapings consistent with $C$. barnesi nematocysts, some experienced only minor symptoms, while in others, envenomation proved fatal (186). Typically, an IS presentation includes a mild local reaction followed by a characteristic incubation period of five to $60 \mathrm{~min}$ before the onset of systemic effects (Figure 2) (191, 192). Pain in the abdomen, chest, lower back, limbs, and joints, is severe, often intractable to opioids and accompanied by extreme distress and agitation (193, 194). In parallel, the manifestation of tachycardia, hypertension, diaphoresis, dyspnea, and in some instances, priapism may occur $(193,194)$. In severe cases, life-threatening complications, such as cardiomyopathy and cardiogenic shock, can arise (193). Due to the significant cardiac dysfunction associated with C. barnesi envenomation, cardiogenic pulmonary oedema may develop $(195,196)$. Tragically, venom-induced intracerebral hemorrhage resulted in the death of two individuals in $2002(192,197)$.

An "Irukandji" antivenom is unavailable, and, as C. barnesi is only one of several causative species, an antivenom is unlikely to be produced (198). Therefore, treatment of severe envenomation is heavily reliant upon opioid-based pain management and symptomatic supportive care, with a mean expected hospital stay of 1.6 days (186). The clinical manifestations of IS have been attributed to excessive catecholamine release, such as seen in pheochromocytoma, scorpionism, or funnel-web spider envenomation $(149,198,199)$. As such, IS has been described as "a painful hypercatecholaminergic condition" (193). Accordingly, individuals at particular risk of fatal outcomes are those with pre-existing cardiovascular pathologies, potentially making the use of alpha/beta-adrenergic blocking agents prohibitive (200).

Supporting this hypothesis, adrenaline and noradrenaline have been transiently detected in the plasma of $C$. barnesi experimentally envenomed piglets (200). Peak catecholamine release was observed $10 \mathrm{~min}$ post intravenous (IV) venom administration, coinciding with the onset of systemic and pulmonary hypertension and tachycardia (200). Plasma 


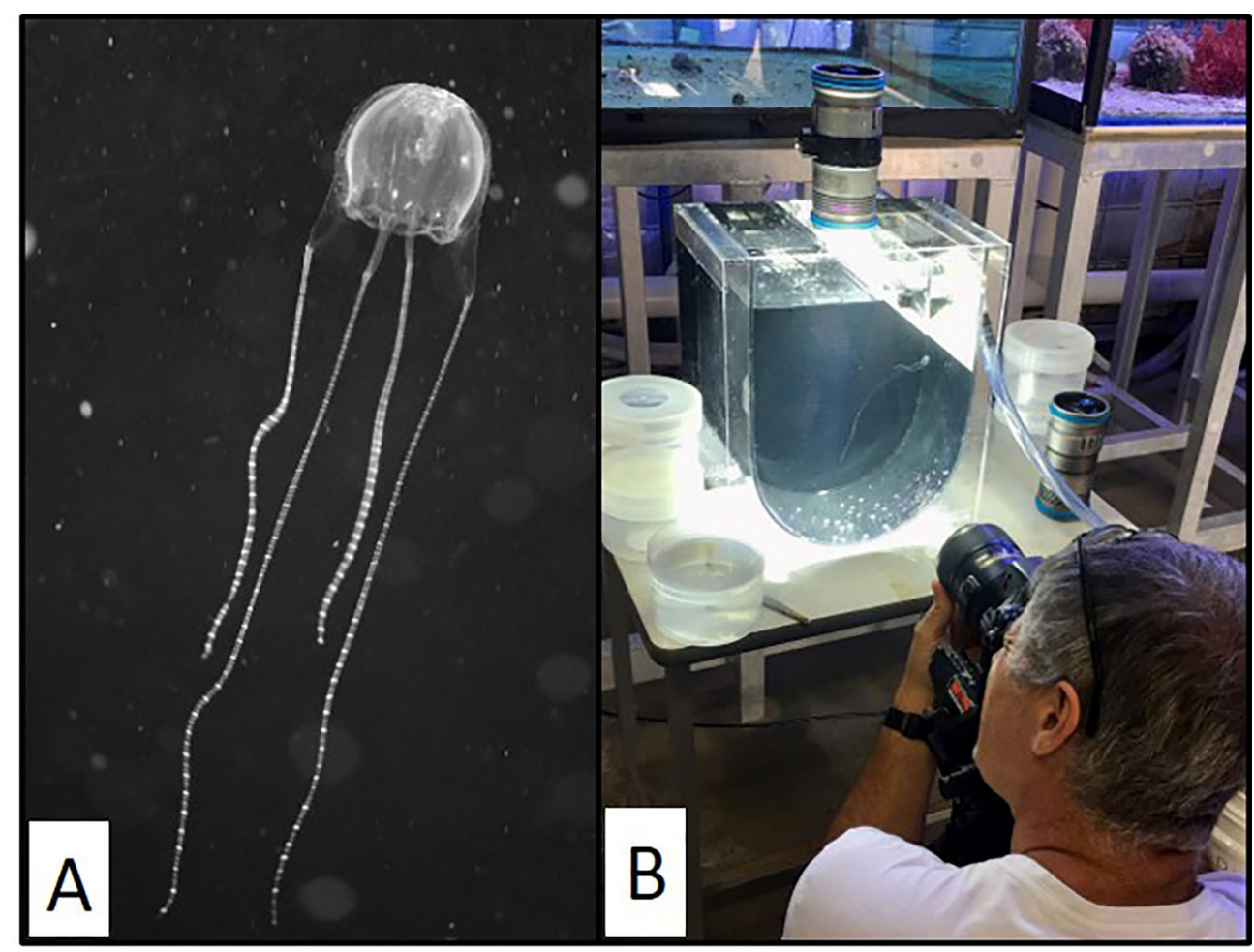

FIGURE 1 Image of Carukia barnesi jellyfish. Images showing (A) close up and (B) relative size of adult C. barnesi jellyfish. Prof Jamie Seymour pictured. Photos were taken by (A) Jamie Seymour (JCU, Cairns, Australia) and (B) Rachael Ryan (JCU, Cairns, Australia).

catecholamines remained elevated in envenomed animals until $60 \mathrm{~min}$ but declined to non-significant levels within $2 \mathrm{~h}$ (200). Pretreatment with $1 \mu \mathrm{mol} / \mathrm{L}$ tetrodotoxin (TTX) attenuated tachycardia responses from rat and guinea-pig isolated right atria but did not significantly alter venom-induced contraction of rat mesenteric small arteries (200). These data suggest the presence of a presynaptic neuronal voltage-gated sodium channel agonist within the venom, as well as the presence of a TTX-insensitive vasoconstrictor (149, 198-200). While a physiological stress response towards IV administration of any toxin may similarly stimulate adrenaline and noradrenaline release, the authors reported the uniqueness of the reaction compared to other box jellyfish venom (200).

Research by Ramasamy et al. found pretreatment with prazosin $(50 \mu \mathrm{g} / \mathrm{kg})$ partially reduced tachycardia in C. barnes $i$ envenomed rats, further supporting the role of endogenous catecholamines in the pathogenesis of IS (198). However, the residual pulse pressure observed in the study suggested the contribution of factors besides catecholamines (198). Furthermore, the result was not reproduced by Winkel et al. with $0.3 \mu \mathrm{M}$ prazosin pretreatment, possibly due to dose- or time-dependent factors that were not clearly stated in either study $(198,200)$. Unfortunately, as both cardiovascular studies required the use of anesthetised animals, euthanised after $2 \mathrm{~h}$, the critical evaluation of later time points was not possible (198). Yet, in sting victims, symptoms can remain for days, potentially requiring intensive care (186).
Regardless, in line with these findings, current clinical guidelines recommend magnesium sulphate $\left(\mathrm{MgSO}_{4}\right)$ therapy to attenuate pain and suppress excessive catecholamine release in severe IS (201). Its success in doing so has generated divided opinions (202). The results of a randomised trial completed in 2012 , and reviewed in 2017, were unable to confirm the ability of $\mathrm{MgSO}_{4}$ infusion to reduce opioid requirement $(202,203)$. Both studies reported varied success from the 39 patients, ultimately showing no significant benefit from $\mathrm{MgSO}_{4}$ therapy $(202,203)$.

Akin to scorpion envenomation, the symptoms of IS cannot be wholly attributed to sympathetic hyperstimulation (10). Also akin to scorpionism, generalised IS symptoms resemble those of CRS. Interestingly, $\mathrm{MgSO}_{4}$ potently suppresses $\mathrm{MNC}$-mediated cytokine production following TLR stimulation (204). $\mathrm{MgSO}_{4}$ increases I $\mathrm{KB} \alpha$ levels in MNCs, thereby decreasing NF- $\kappa \mathrm{B}$ nuclear translocation and its activity (204). Accordingly, the ability of $\mathrm{MgSO}_{4}$ to inhibit pain in some sting patients could in part be due to a dampened immune response, although this theory has not been investigated.

Presently, neither catecholamines nor inflammatory mediators have been measured in C. barnesi sting patients. Recently, a study by Staedtke et al. proposed an intriguing link between "cytokine storm" and "catecholamine storm" in systemic inflammatory response syndrome (SIRS) and capillary leak syndrome, which may apply to venom-induced systemic inflammation $(205,206)$. This study showed that adrenaline 


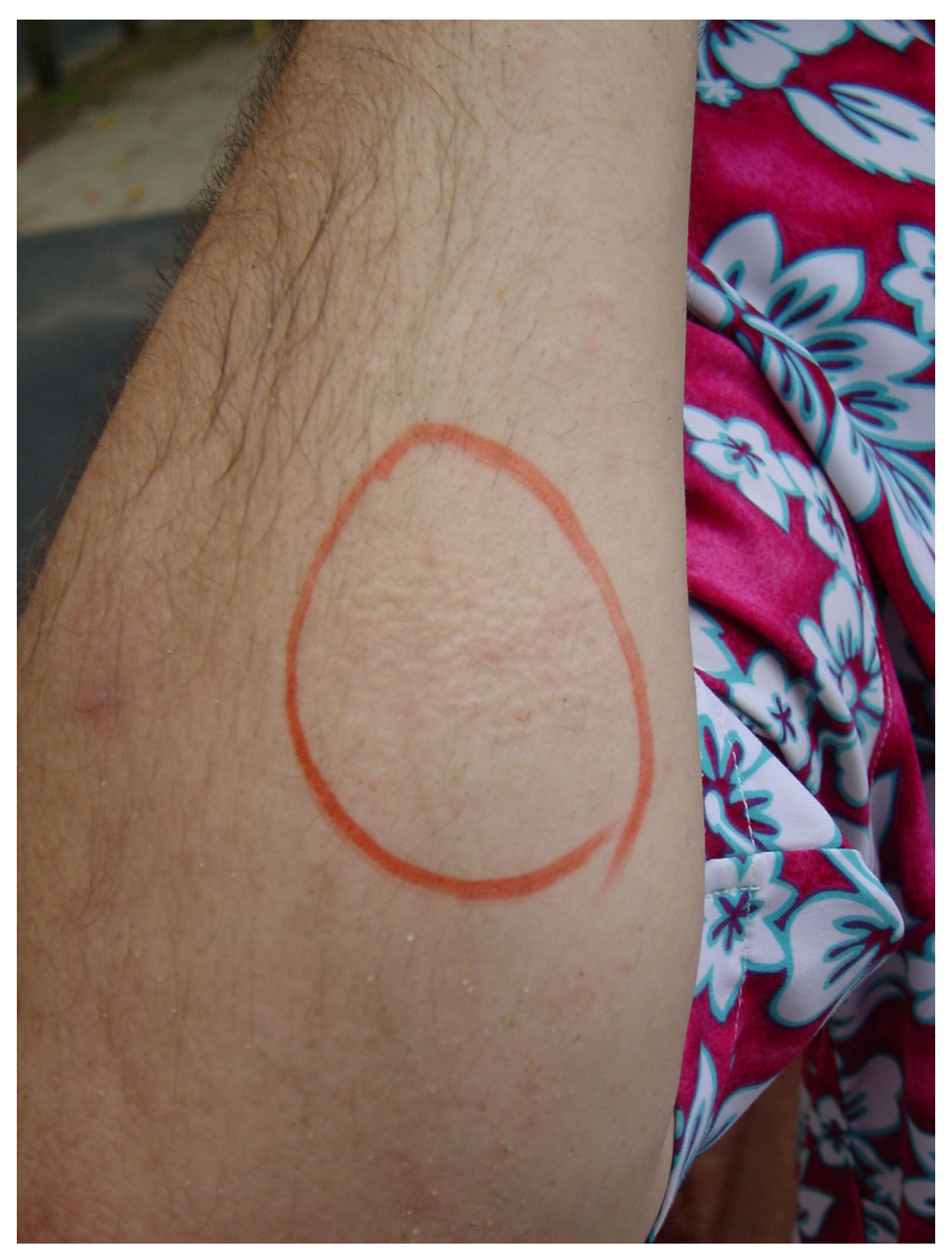

FIGURE 2 | Local response to C. barnesi envenomation. Image showing a typical dermal reaction on the arm following a sting from a C. barnesi jellyfish. The red marker indicates the sting site. Photo by Jamie Seymour (JCU, Cairns, Australia).

contributes to the positive feed-forward cytokine dysregulation seen in CRS (206). Encouragingly, the blockade of $\alpha_{1}$-adrenergic receptors (also expressed on immune cells) or the inhibition of tyrosine hydroxylase (required for catecholamine biosynthesis) by prazosin or metyrosine hindered the self-amplifying proinflammatory loop in vitro and in vivo (206).

Specifically, CRS, induced by humanised CD19 CAR-T cells in mice engrafted with a leukemia cell line, caused excessive levels of adrenaline, noradrenaline, and myeloid-derived cytokines (IL-6, KC, MCP-1, and TNF) in the plasma of animals with high tumour burdens (206). Consequently, higher mortality was observed among these mice. In contrast, pretreatment with metyrosine or prazosin lowered circulating catecholamines and cytokines, improving survival (206). In mouse peritoneal MФs, LPS-stimulation induced the release of catecholamines and proinflammatory cytokines, IL-6, KC, MCP1 , and TNF (206). Cytokine and catecholamine secretion was markedly enhanced in LPS/adrenaline co-cultures (206).
Conversely, reduced $\mathrm{M} \Phi$ catecholamine production significantly reduced IL-6, KC, MCP-1, and TNF levels (206). Although this model is unrelated to envenomation, it suggests that immune stimuli, such as venom-induced TLR activation, initiates a proinflammatory response that is enhanced by the presence of catecholamines. The dual stimulatory signals create a positive feed-forward loop, resulting in cytokine amplification. Collectively, these studies suggest a therapeutic potential of prazosin for severe IS, except where contraindicated, and warrant the investigation of plasma cytokines in sting patients.

However, while such research supports the therapeutic inhibition of catecholamines in envenomation, understanding their possible protective function has not been investigated. For example, it is known that catecholamine release promotes alveolar fluid clearance (174). In scorpionism, a rapid catecholamine surge following a dangerous sting is reasoned to increase alveolar fluid reabsorption, protecting the lungs from venom-induced flooding (174). Therefore, the resolution of the 
surge may decrease the ability of the lungs to clear venominduced oedema, ultimately progressing toward fatal pulmonary oedema (174). Accordingly, animal models of IS should thoroughly scrutinise both the benefits and limitations of these mediators.

Finally, given the immune system's propensity toward hyperresponsiveness and allergic reaction to jellyfish venom, the overlap in IS and CRS generalised symptoms, and the recently described link between CRS and catecholamine storm, immune involvement in the pathology is plausible. Nevertheless, to date, no $C$. barnesi immunology-based research has been published.

\section{THE IMMUNOSUPPRESSIVE POTENTIAL OF VENOM-DERIVED MOLECULES}

Despite the health burden of human envenomation, venomimmune interactions have been exploited in traditional medicine for centuries (207). More recently, research groups throughout the world have demonstrated the in vitro and in vivo efficacy of whole venom and venom-derived compounds in ameliorating a wide range of autoimmune symptoms (112, 208-211).

Presently, the most promising drug leads belong to the class of ion channel modulators. Ion channels, particularly calciumactivated and voltage-gated potassium channels, are attractive therapeutic targets for autoimmune diseases. Firstly, ion channels, such as the Shaker-related voltage-gated $\mathrm{K}_{\mathrm{V}} 1.3$ and $C$ $A_{-}^{2+}$ - dependent $\mathrm{KCa} 3.1$, regulate $\mathrm{Ca}^{2+}$ signalling in activated immune cells, allowing cell depolarisation and maintenance of membrane potential. Intracellular $\mathrm{Ca}^{2+}$ levels dictate $\mathrm{T}$ cell activation, proliferation, metabolism and cytokine production (212). Secondly, unique ion channel dimers are differentially expressed in various tissues, including immune cell subsets, permitting cell type and subset-specific blockade $(213,214)$. For example, activated effector memory $\mathrm{T}$ cells $\left(\mathrm{T}_{\mathrm{EM}}\right), \mathrm{B}$ cells and MФs, known mediators in the pathogenesis of various autoimmune diseases, preferentially upregulate $\mathrm{K}_{\mathrm{V}} 1.3$ (215217). In contrast, naïve $\left(T_{n}\right)$ and central memory cells $\left(T_{C M}\right)$ express $\mathrm{KCa} 3.1$ ion channels, allowing for channel-specific inhibition (218). Finally, inhibition of $\mathrm{Ca}^{2+}$ influx via ion channel blockade allows targeted and reversible immune modulation, rather than complete $\mathrm{T}$ cell suppression, as induced by $\mathrm{T}$ cell $\mathrm{Ca}^{2+}$ modulating drugs, including calcineurin inhibitors and steroids.

Venom from snakes, spiders, scorpions, cone snails, and sea anemones comprise a diverse range of peptide and small molecule ion channel blockers that exhibit selectivity at picomolar concentrations (219). Blockade of lymphocyte ion channels using venom-derived compounds has therapeutic effects in animal models of rheumatoid arthritis (RA), asthma, multiple sclerosis (MS), delayed-type hypersensitivity, and allograft rejection $(113,208,219-224)$. Notably, a selective peptide blocker, Stichodactyla helianthus toxin (ShK), from sea anemone venom, and anuroctoxin, a peptidyl toxin isolated from Buthus sindicus scorpion venom, have been shown to specifically target $\mathrm{K}_{\mathrm{V}} 1.3$ channels with high affinity, preventing $\mathrm{Ca}^{2+}$ influx and thereby inhibiting $\mathrm{T}_{\mathrm{EM}}$ activation, proliferation and cytokine production $(113,115,225-227)$.

Structural studies centred on the selectivity of peptide ion channel blockers have revealed that specificity is due to single amino acid effects rather than en bloc backbone structure (228). Thus, venom-derived peptides may act as promising drug scaffolds, notably because disulphide bonds encode robust biological stability (229). This has important implications for drug development, as synthetic manipulation may improve drug activity or remove toxicity from the natural peptide blueprint. For example, ShK(L5), a synthetic analog of ShK, contains an Nterminal L-phosphotyrosine extension and shows higher selectivity than the native peptide for $\mathrm{K}_{\mathrm{V}} 1.3$ channels over the neuronal ion channel $\mathrm{K}_{\mathrm{V}} 1.1$ (113).

Aside from ion channel blockade, venom-derived components have demonstrated potent in vitro and in vivo anti-inflammatory activity through the cholinergic antiinflammatory pathway via an alpha7 nicotinic acetylcholine receptor antagonist (114). Venom-derived peptides, such as the $\alpha$-neurotoxin from the Thailand cobra, are potent nicotinic receptor antagonists (218). In a rodent RA model, Cobratoxintreatment reduced expression of the pro-inflammatory cytokines IL-1 $\beta$, IL-2, and TNF $\alpha$, resulting in decreased paw sensitivity and joint destruction (230).

Other neurotoxins, such as the principal toxin (NTX) from Naja atra venom (NNAV), have shown therapeutic effects in animal models of adjunctive arthritis, RA, Systemic Lupus Erythematosus (SLE), and nephropathy (209, 211). Additionally, NTX-treatment prolonged skin allograft survival in rats and inhibited cell-mediated immune responses in a dose-dependent manner through decreased Th1-type cytokines (IL-2 and IFN- $\gamma$ ). Although low NTX concentrations were cytotoxic, heat-treatment reduced NTX toxicity without reducing its immunosuppressive activity (211). In another study, orally administered NTX suppressed murine $\mathrm{T}$ cell proliferation, specifically Th17 and $\mathrm{CD} 8^{+} \mathrm{T}$ cell activity, increasing NK cell and B cell proliferation in a dose-dependent manner (209).

Venom from the honey bee has been used for centuries in traditional medicine to treat chronic inflammatory diseases due to its reported anti-inflammatory activity (207). Investigations into the mechanism of action of honey bee venom and its major components, melittin and phospholipase A2, have confirmed a protective effect in animal models of asthma and RA $(207,231)$. The polarisation of T cells towards a Th2 phenotype is associated with allergies and chronic inflammatory diseases (232). An essential driving factor in lineage determination is cytokine expression. It has been shown that melittin inhibits LPSinduced inflammation by binding to the $\mathrm{C}$ - terminus of the $\mathrm{NF}-\kappa \mathrm{B}$ p50 subunit, thus preventing translocation into the nucleus and transcription of pro-inflammatory cytokines, including TNF (233-235). Moreover, treatment with whole honey bee venom polarised $\mathrm{T}$ cells towards a Th1 phenotype by inducing T-bet and IFN- $\gamma$ in $\mathrm{CD}^{+} \mathrm{T}$ cells (210). Conversely, $\mathrm{PLA}_{2}$, an enzyme found within the venom of multiple species, including the western honey bee, can hydrolyze membrane 

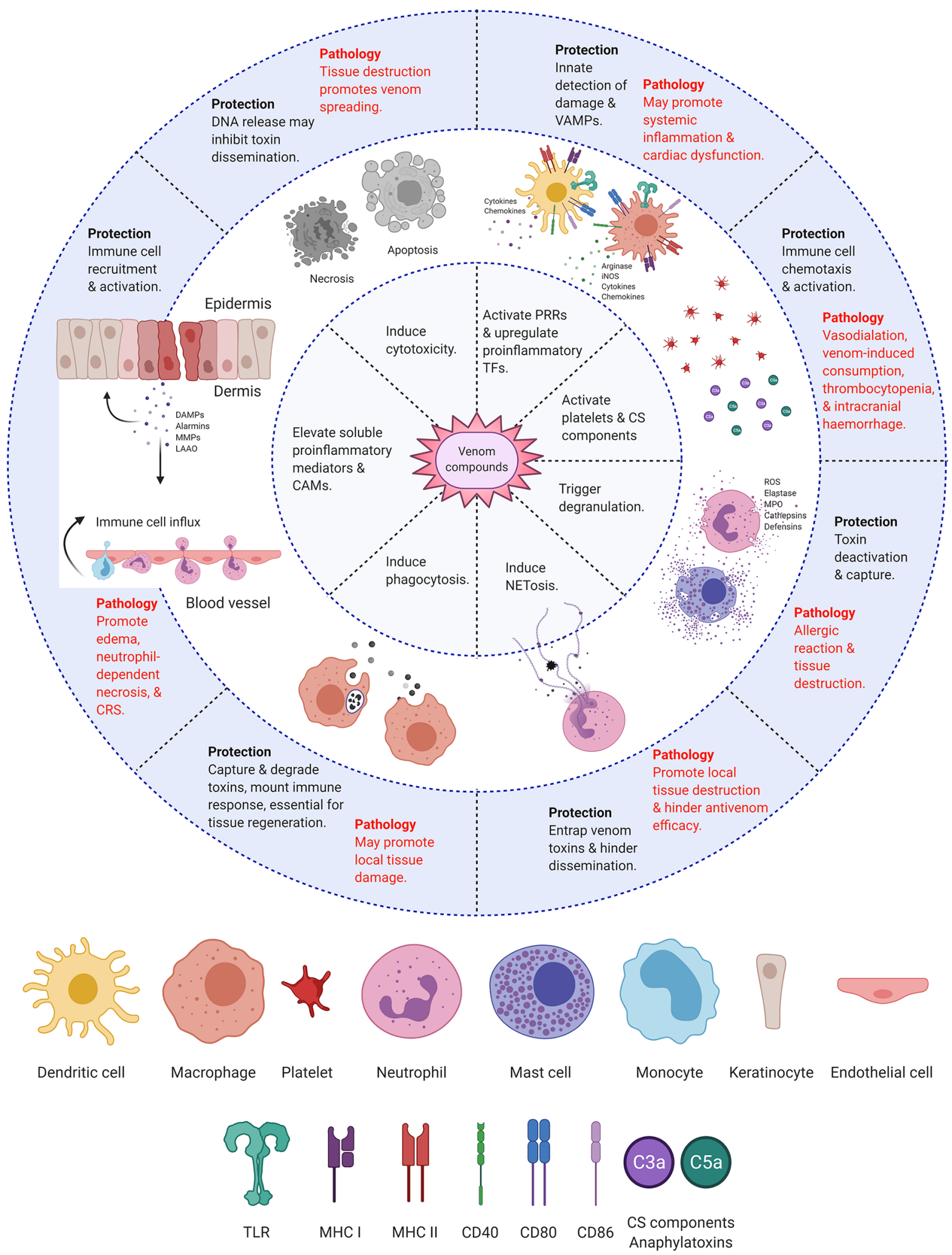

FIGURE 3 | Immunological responses to envenomation. Diagram summarizing the protective and pathological responses of the host's immune system towards venom compounds. Created with BioRender.com. 
phospholipids and induce Th2 cytokine responses through the activation of ST2, a component of the IL-33 receptor on innate immune cells (236).

Other known venom immune modulators include tick salivary protein (Salp) 15 from Ixodes scapularis and spermine. Salp15 binds the CD4 co-receptor, MHC-II, inhibiting TCR ligation and $\mathrm{T}$ cell activation by misaligning CD4 with the TCR complex (237). Spermine, an acylpolyamine found in snake and spider venom, suppresses mitogen-induced activation and proliferation of PBMCs by inhibiting LAF-1 protein expression, involved in RNA remodelling (238).

Collectively, these studies highlight the potential of venomderived molecules to modulate immune cells as unmodified venom-derived compounds or as scaffolds for drug development. Venom-derived compounds induce immune suppression using diverse modes of action. Thus, screening venom for its immunosuppressive and immune-activating potential may result in new immunomodulatory drugs and the discovery of new biological pathways.

\section{CONCLUSION}

Conferring protection against venom's potentially lethal action requires rapid immune recognition and response. Extensive research focuses on the degree to which immune responses themselves contribute to the severity of envenomation (Figure 3). However, there is disagreement regarding whether the body's defensive reactions are helpful or harmful. Perhaps the most significant cause of division lies in the difficulty of distinguishing the actual venom-induced symptoms from immune-induced pathology. The classic inflammation markers (heat, pain, redness, swelling, and loss of function) are typical biological responses to envenomation across many species. Therefore, determining which symptoms

\section{REFERENCES}

1. Jouiaei M, Yanagihara AA, Madio B, Nevalainen TJ, Alewood PF, Fry BG. Ancient Venom Systems: A Review on Cnidaria Toxins. Toxins (2015) 7 (6):2251-71. doi: 10.3390/toxins7062251

2. Touchard A, Aili SR, Fox EGP, Escoubas P, Orivel J, Nicholson GM, et al. The Biochemical Toxin Arsenal From Ant Venoms. Toxins (2016) 8(1):30. doi: 10.3390/toxins 8010030

3. Chan YS, Cheung RC, Xia L, Wong JH, Ng TB, Chan WY. Snake Venom Toxins: Toxicity and Medicinal Applications. Appl Microbiol Biotechnol (2016) 100(14):6165-81. doi: 10.1007/s00253016-7610-9

4. King G, Hardy M. Spider-Venom Peptides: Structure, Pharmacology, and Potential for Control of Insect Pests. Annu Rev Entomol (2012) 58:475-96. doi: 10.1146/annurev-ento-120811-153650

5. Gutiérrez JM, Escalante T, Rucavado A, Herrera C, Fox JW. A Comprehensive View of the Structural and Functional Alterations of Extracellular Matrix by Snake Venom Metalloproteinases (Svmps): Novel Perspectives on the Pathophysiology of Envenoming. Toxins (2016) 8 (10):304. doi: 10.3390/toxins8100304

6. Chippaux JP, Goyffon M. Epidemiology of Scorpionism: A Global Appraisal. Acta Trop (2008) 107(2):71-9. doi: 10.1016/j.actatropica. 2008.05.021 are treatable using immunological approaches requires further research.

Nevertheless, venom's ability to modulate immune activity has two therapeutic implications. Firstly, continued research could inform improved treatment strategies for fatal bites and stings. Secondly, as venom is a rich source of specific and potent biomodulators, exploring venom-immune interactions may lead to discovering novel pathways/receptors or the development of venom-derived immunomodulatory drugs.

\section{AUTHOR CONTRIBUTIONS}

Writing-original draft preparation, RR. Writing-review and editing, RR, JM, MI, JS, AL, and JL. Supervision, JM, MI, and JL. Funding acquisition, JM. All authors contributed to the article and approved the submitted version.

\section{FUNDING}

This research received funding from the Australian National Health and Medical Council (NHMRC) (1031652 \& 1131732). RR was supported by an Australian Government Research Training Program stipend. MI is supported by the TALENTO Program by the Regional Madrid Government (2018-T1/BIO11262). JM (Career Development Fellowship APP1131732) is supported by the Australian National Health \& Medical Research Council.

\section{ACKNOWLEDGMENTS}

I would very much like to thank Dr. Paul Giacomin for critical reading of the original draft manuscript.

7. Helbling A, Müller UR. Allergic Reactions to Stinging and Biting Insects, Fifth ed. R Rich, T Fleisher, W Shearer, H Schroeder, A Frew and C Weyand, editors. London: Elsevier (2019). pp. 601-10.e1.

8. Egawa G, Kabashima K. Skin as a Peripheral Lymphoid Organ: Revisiting the Concept of Skin-Associated Lymphoid Tissues. J Invest Dermatol (2011) 131(11):2178-85. doi: 10.1038/jid.2011.198

9. Eyerich S, Eyerich K, Traidl-Hoffmann C, Biedermann T. Cutaneous Barriers and Skin Immunity: Differentiating a Connected Network. Trends Immunol (2018) 39(4):315-27. doi: 10.1016/j.it.2018.02.004

10. Reis MB, Zoccal KF, Gardinassi LG, Faccioli LH. Scorpion Envenomation and Inflammation: Beyond Neurotoxic Effects. Toxicon Off J Int Soc Toxinol (2019) 167:174-9. doi: 10.1016/j.toxicon.2019.06.219

11. Aristizábal B GÁ. Innate Immune System. Autoimmunity: From Bench to Bedside. SY Anaya JM and A Rojas-Villarraga, editors. Bogota (Colombia: El Rosario University Press (2013).

12. Mogensen TH. Pathogen Recognition and Inflammatory Signaling in Innate Immune Defenses. Clin Microbiol Rev (2009) 22(2):240-73. doi: 10.1128/ CMR.00046-08

13. Strbo N, Yin N, Stojadinovic O. Innate and Adaptive Immune Responses in Wound Epithelialization. Adv Wound Care (New Rochelle) (2014) 3(7):492501. doi: 10.1089/wound.2012.0435

14. Dunkelberger JR, Song W-C. Complement and its Role in Innate and Adaptive Immune Responses. Cell Res (2010) 20(1):34-50. doi: 10.1038/cr.2009.139 
15. Palm NW, Medzhitov R. Role of the Inflammasome in Defense Against Venoms. Proc Natl Acad Sci U States America (2013) 110(5):1809-14. doi: 10.1073/pnas.1221476110

16. Teixeira CF, Zamuner SR, Zuliani JP, Fernandes CM, Cruz-Hofling MA, Fernandes I, et al. Neutrophils do Not Contribute to Local Tissue Damage, But Play a Key Role in Skeletal Muscle Regeneration, in Mice Injected With Bothrops Asper Snake Venom. Muscle Nerve (2003) 28(4):449-59. doi: 10.1002/mus.10453

17. Xu H, Timares L, Elmets CA. Host Defenses in the Skin. In: RR Rich, TA Fleisher, WT Shearer, HW Schroeder, AJ Frew and CM Weyand, editors. Clinical Immunology. Elsevier Saunders (2013). p. 228-38.

18. Schlüter H, Upjohn E, Varigos G, Kaur P. Burns and Skin Ulcers. In: R Lanza and A Atala, editors. Essentials of Stem Cell Biology, 3rd ed. Boston: Academic Press (2014). p. 501-13.

19. Albanesi C, Madonna S, Gisondi P, Girolomoni G. The Interplay Between Keratinocytes and Immune Cells in the Pathogenesis of Psoriasis. Front Immunol (2018) 9:1549-. doi: 10.3389/fimmu.2018.01549

20. Pivarcsi A, Kemeny L, Dobozy A. Innate Immune Functions of the Keratinocytes. A Rev Acta Microbiol Immunol Hungarica (2004) 51 (3):303-10. doi: 10.1556/AMicr.51.2004.3.8

21. Lebre MC, van der Aar AMG, van Baarsen L, van Capel TMM, Schuitemaker JHN, Kapsenberg ML, et al. Human Keratinocytes Express Functional TollLike Receptor 3, 4, 5, and 9. J Invest Dermatol (2007) 127(2):331-41. doi: 10.1038/sj.jid.5700530

22. Costal-Oliveira F, Stransky S, Guerra-Duarte C, Naves de Souza DL, VivasRuiz DE, Yarlequé A, et al. L-Amino Acid Oxidase From Bothrops Atrox Snake Venom Triggers Autophagy, Apoptosis and Necrosis in Normal Human Keratinocytes. Sci Rep (2019) 9(1):781-. doi: 10.1038/s41598-018$37435-4$

23. Stransky S, Costal-Oliveira F, Lopes-de-Souza L, Guerra-Duarte C, ChávezOlórtegui C, Braga VMM. In Vitro Assessment of Cytotoxic Activities of Lachesis Muta Muta Snake Venom. PloS neglected Trop Dis (2018) 12(4): e0006427. doi: 10.1371/journal.pntd.0006427

24. Silva-de-Franca F, Villas-Boas IM, Serrano SMT, Cogliati B, Chudzinski SAA, Lopes PH, et al. Naja Annulifera Snake: New Insights Into the Venom Components and Pathogenesis of Envenomation. PloS Negl Trop Dis (2019) 13(1):e0007017. doi: 10.1371/journal.pntd.0007017

25. Bhattacharjee P, Mitra J, Bhattacharyya D. L-Amino Acid Oxidase From Venoms. Dordrecht: Springer Netherlands (2017). p. 295-320.

26. Paixão-Cavalcante D, van den Berg CW, de Freitas Fernandes-Pedrosa M, Gonçalves de Andrade RM, Tambourgi DV. Role of Matrix Metalloproteinases in HaCaT Keratinocytes Apoptosis Induced by Loxosceles Venom Sphingomyelinase D. J Invest Dermatol (2006) 126 (1):61-8. doi: 10.1038/sj.jid.5700049

27. Telser A. Molecular Biology of the Cell. Shock. 18, 4th ed. New York: Garland Science (2002). p. 289. Available from: https://www.ncbi.nlm.nih.gov/books/ NBK21054/.

28. Al-Soudi A, Kaaij MH, Tas SW. Endothelial Cells: From Innocent Bystanders to Active Participants in Immune Responses. Autoimmun Rev (2017) 16(9):951-62. doi: 10.1016/j.autrev.2017.07.008

29. Khakpour S, Wilhelmsen K, Hellman J. Vascular Endothelial Cell Toll-like Receptor Pathways in Sepsis. Innate Immun (2015) 21(8):827-46. doi: $10.1177 / 1753425915606525$

30. Pugin J, Ulevitch RJ, Tobias PS. Tumor Necrosis Factor-Alpha and Interleukin-1 Beta Mediate Human Endothelial Cell Activation in Blood At Low Endotoxin Concentrations. J Inflammation (1995) 45(1):49-55.

31. Krishnaswamy G, Kelley J, Yerra L, Smith JK, Chi DS. Human Endothelium as a Source of Multifunctional Cytokines: Molecular Regulation and Possible Role in Human Disease. J Interferon Cytokine Res (1999) 19(2):91-104. doi: $10.1089 / 107999099314234$

32. Teijaro John R, Walsh Kevin B, Cahalan S, Fremgen Daniel M, Roberts E, Scott F, et al. Endothelial Cells are Central Orchestrators of Cytokine Amplification During Influenza Virus Infection. Cell (2011) 146(6):98091. doi: 10.1016/j.cell.2011.08.015

33. Pober JS, Merola J, Liu R, Manes TD. Antigen Presentation by Vascular Cells. Front Immunol (2017) 8:1907. doi: 10.3389/fimmu.2017.01907

34. Joyce DE, Nelson DR, Grinnell BW. Leukocyte and Endothelial Cell Interactions in Sepsis: Relevance of the Protein C Pathway. Crit Care Med (2004) 32(5 Suppl):S280-6. doi: 10.1097/01.CCM.0000128037.72072.22
35. Nourshargh S, Alon R. Leukocyte Migration Into Inflamed Tissues. Immunity (2014) 41(5):694-707. doi: 10.1016/j.immuni.2014.10.008

36. Krieglstein CF, Granger DN. Adhesion Molecules and Their Role in Vascular Disease. Am J Hypertens (2001) 14(6 Pt 2):44s-54s. doi: 10.1016/ S0895-7061(01)02069-6

37. Danese S, Dejana E, Fiocchi C. Immune Regulation by Microvascular Endothelial Cells: Directing Innate and Adaptive Immunity, Coagulation, and Inflammation. J Immunol (Baltimore Md 1950) (2007) 178(10):6017-22. doi: 10.4049/jimmunol.178.10.6017

38. Borkow G, Lomonte B, Gutierrez JM, Ovadia M. Effect of Various Viperidae and Crotalidae Snake Venoms on Endothelial Cells In Vitro. Toxicon Off $J$ Int Soc Toxinol (1994) 32(12):1689-95. doi: 10.1016/0041-0101(94)90330-1

39. Nowatzki J, de Sene RV, Paludo KS, Veiga SS, Oliver C, Jamur MC, et al. Brown Spider Venom Toxins Interact With Cell Surface and are Endocytosed by Rabbit Endothelial Cells. Toxicon Off J Int Soc Toxinol (2010) 56(4):535-43. doi: 10.1016/j.toxicon.2010.03.027

40. Chung $\mathrm{C}-\mathrm{H}, \mathrm{Wu} \mathrm{W}-\mathrm{B}$, Huang T-F. Aggretin, a Snake Venom-Derived Endothelial Integrin $\alpha 2 \beta 1$ Agonist, Induces Angiogenesis Via Expression of Vascular Endothelial Growth Factor. Blood (2004) 103(6):2105-13. doi 10.1182/blood-2003-07-2483

41. Paludo KS, Gremski LH, Veiga SS, Chaim OM, Gremski W, de Freitas Buchi D, et al. The Effect of Brown Spider Venom on Endothelial Cell Morphology and Adhesive Structures. Toxicon Off J Int Soc Toxinol (2006) 47(8):844-53. doi: 10.1016/j.toxicon.2006.02.006

42. Delafontaine M, Villas-Boas IM, Mathieu L, Josset P, Blomet J, Tambourgi DV. Enzymatic and Pro-Inflammatory Activities of Bothrops Lanceolatus Venom: Relevance for Envenomation. Toxins (2017) 9(8):244. doi: 10.3390/ toxins 9080244

43. Patel KD, Modur V, Zimmerman GA, Prescott SM, McIntyre TM. The Necrotic Venom of the Brown Recluse Spider Induces Dysregulated Endothelial Cell-Dependent Neutrophil Activation. Differential Induction of GM-CSF, Il-8, and E-selectin Expression. J Clin Invest (1994) 94(2):63142. doi: 10.1172/JCI117379

44. Chiu S, Bharat A. Role of Monocytes and Macrophages in Regulating Immune Response Following Lung Transplantation. Curr Opin Organ Transplant (2016) 21(3):239-45. doi: 10.1097/MOT.0000000000000313

45. Randolph GJ, Jakubzick C, Qu C. Antigen Presentation by Monocytes and Monocyte-Derived Cells. Curr Opin Immunol (2008) 20(1):52-60. doi: 10.1016/j.coi.2007.10.010

46. Franken L, Schiwon M, Kurts C. Macrophages: Sentinels and Regulators of the Immune System. Cell Microbiol (2016) 18(4):475-87. doi: 10.1111/ cmi. 12580

47. Ma WT, Gao F, Gu K, Chen DK. The Role of Monocytes and Macrophages in Autoimmune Diseases: A Comprehensive Review. Front Immunol (2019) 10:1140. doi: 10.3389/fimmu.2019.01140

48. Sampaio SC, Sousa-e-Silva MC, Borelli P, Curi R, Cury Y. Crotalus Durissus Terrificus Snake Venom Regulates Macrophage Metabolism and Function. J Leukocyte Biol (2001) 70(4):551-8.

49. Setubal SS, Pontes AS, Furtado JL, Kayano AM, Stábeli RG, Zuliani JP. Effect of Bothrops Alternatus Snake Venom on Macrophage Phagocytosis and Superoxide Production: Participation of Protein Kinase C. J Venomous Anim Toxins Including Trop Dis (2011) 17(4):430-41. doi: 10.1590/S167891992011000400010

50. Saadi S, Assarehzadegan MA, Pipelzadeh MH, Hadaddezfuli R. Induction of IL-12 From Human Monocytes After Stimulation With Androctonus Crassicauda Scorpion Venom. Toxicon Off J Int Soc Toxinol (2015) 106:117-21. doi: 10.1016/j.toxicon.2015.09.029

51. Pires WL, Kayano AM, Castro OB, Paloschi MV, Lopes JA, Boeno CN, et al. Lectin Isolated From Bothrops Jararacussu Venom Induces IL-10 Release by TCD4+ Cells and TNF- $\alpha$ Release by Monocytes and Natural Killer Cells. J leukocyte Biol (2019) 106(3):595-605. doi: 10.1002/JLB.MA1118-463R

52. Rojas JM, Aran-Sekul T, Cortes E, Jaldín R, Ordenes K, Orrego PR, et al. Phospholipase D From Loxosceles Laeta Spider Venom Induces IL-6, Il-8, Cxcl1/Gro- $\alpha$, and CCL2/MCP-1 Production in Human Skin Fibroblasts and Stimulates Monocytes Migration. Toxins (2017) 9(4):125. doi: 10.3390/ toxins 9040125

53. Zoccal KF, Ferreira GZ, Prado MKB, Gardinassi LG, Sampaio SV, Faccioli LH. LTB4 and PGE2 Modulate the Release of MIP-1 $\alpha$ and IL- $1 \beta$ by Cells 
Stimulated With Bothrops Snake Venoms. Toxicon Off J Int Soc Toxinol (2018) 150:289-96. doi: 10.1016/j.toxicon.2018.06.066

54. Khemili D, Valenzuela C, Laraba-Djebari F, Hammoudi-Triki D. Differential Effect of Androctonus Australis Hector Venom Components on Macrophage KV Channels: Electrophysiological Characterization. Eur Biophys J (2019) 48(1):1-13. doi: 10.1007/s00249-018-1323-1

55. Hsu C-C, Chuang W-J, Chung C-H, Chang C-H, Peng H-C, Huang T-F. Snake Venom Disintegrin Inhibits the Activation of Toll-Like Receptors and Alleviates Sepsis Through Integrin alphaVbeta3 Blockade. Sci Rep (Nature Publisher Group) (2016) 6:23387. doi: 10.1038/srep23387

56. Zoccal KF, Bitencourt C, Paula-Silva FWG, CA S, de Castro Figueiredo Bordon K, Arantes EC, et al. Tlr2, TLR4 and CD14 Recognize VenomAssociated Molecular Patterns From Tityus Serrulatus to Induce Macrophage-Derived Inflammatory Mediators. PloS One (2014) 9(2): e88174-e. doi: 10.1371/journal.pone.0088174

57. Veda P. Why are Neutrophils Polymorphonuclear? Eur J Inflammation (2011) 9(2):85-93. doi: 10.1177/1721727X1100900201

58. Kruger P, Saffarzadeh M, Weber ANR, Rieber N, Radsak M, von Bernuth H, et al. Neutrophils: Between Host Defence, Immune Modulation, and Tissue Injury. PloS Pathog (2015) 11(3):e1004651. doi: 10.1371/journal.ppat.1004651

59. Summers C, Rankin SM, Condliffe AM, Singh N, Peters AM, Chilvers ER. Neutrophil Kinetics in Health and Disease. Trends Immunol (2010) 31 (8):318-24. doi: 10.1016/j.it.2010.05.006

60. Pillay J, den Braber I, Vrisekoop N, Kwast LM, de Boer RJ, Borghans JA, et al. In Vivo Labeling With $2 \mathrm{H} 2 \mathrm{O}$ Reveals a Human Neutrophil Lifespan of 5.4 days. Blood (2010) 116(4):625-7. doi: 10.1182/blood-2010-01-259028

61. Tecchio C, Micheletti A, Cassatella MA. Neutrophil-Derived Cytokines: Facts Beyond Expression. Front Immunol (2014) 5:508-. doi: 10.3389/ fimmu.2014.00508

62. Rorvig S, Ostergaard O, Heegaard NH, Borregaard N. Proteome Profiling of Human Neutrophil Granule Subsets, Secretory Vesicles, and Cell Membrane: Correlation With Transcriptome Profiling of Neutrophil Precursors. J leukocyte Biol (2013) 94(4):711-21. doi: 10.1189/jlb.1212619

63. Papayannopoulos V. Neutrophil Extracellular Traps in Immunity and Disease. Nat Rev Immunol (2018) 18(2):134-47. doi: 10.1038/nri.2017.105

64. Kaplan MJ, Radic M. Neutrophil Extracellular Traps: Double-edged Swords of Innate Immunity. J Immunol (Baltimore Md 1950) (2012) 189(6):2689-95. doi: 10.4049/jimmunol.1201719

65. Katkar GD, Sundaram MS, NaveenKumar SK, Swethakumar B, Sharma RD, Paul M, et al. Netosis and Lack of DNase Activity are Key Factors in Echis Carinatus Venom-Induced Tissue Destruction. Nat Commun (2016) 7:11361. doi: 10.1038/ncomms11361

66. Stackowicz J, Balbino B, Todorova B, Godon O, Iannascoli B, Jönsson F, et al. Evidence That Neutrophils do Not Promote Echis Carinatus VenomInduced Tissue Destruction. Nat Commun (2018) 9(1):2304. doi: 10.1038/ s41467-018-04688-6

67. Williams DJ, Faiz MA, Abela-Ridder B, Ainsworth S, Bulfone TC, Nickerson $\mathrm{AD}$, et al. Strategy for a Globally Coordinated Response to a Priority Neglected Tropical Disease: Snakebite Envenoming. PloS Negl Trop Dis (2019) 13(2):e0007059. doi: 10.1371/journal.pntd.0007059

68. Krystel-Whittemore M, Dileepan KN, Wood JG. Mast Cell: A MultiFunctional Master Cell. Front Immunol (2016) 6:620-. doi: 10.3389/ fimmu.2015.00620

69. Galli SJ, Starkl P, Marichal T, Tsai M. Mast Cells and IgE in Defense Against Venoms: Possible "Good Side" of Allergy? Allergology Int Off J Japanese Soc Allergology (2016) 65(1):3-15. doi: 10.1016/j.alit.2015.09.002

70. Rivera J, Gilfillan AM. Molecular Regulation of Mast Cell Activation. J Allergy Clin Immunol (2006) 117(6):1214-25. doi: 10.1016/j.jaci.2006.04.015

71. Moon TC, Befus AD, Kulka M. Mast Cell Mediators: Their Differential Release and the Secretory Pathways Involved. Front Immunol (2014) 5:569-. doi: $10.3389 /$ fimmu.2014.00569

72. Gilfillan AM, Tkaczyk C. Integrated Signalling Pathways for Mast-Cell Activation. Nat Rev Immunol (2006) 6(3):218-30. doi: 10.1038/nri1782

73. Metcalfe DD, Peavy RD, Gilfillan AM. Mechanisms of Mast Cell Signaling in Anaphylaxis. J Allergy Clin Immunol (2009) 124(4):639-48. doi: 10.1016/ j.jaci.2009.08.035

74. Galli SJ, Tsai M. Ige and Mast Cells in Allergic Disease. Nat Med (2012) 18 (5):693-704. doi: 10.1038/nm.2755
75. Galli SJ, Starkl P, Marichal T, Tsai M. Mast Cells and IgE can Enhance Survival During Innate and Acquired Host Responses to Venoms. Trans Am Clin Climatological Assoc (2017) 128:193-221.

76. Pejler G, Rönnberg E, Waern I, Wernersson S. Mast Cell Proteases: Multifaceted Regulators of Inflammatory Disease. Blood (2010) 115 (24):4981-90. doi: 10.1182/blood-2010-01-257287

77. Fehervari Z. Mast Cells Crack Down on Venom. Nat Immunol (2011) 12 (12):1141-. doi: 10.1038/ni.2177

78. Akdis M, Aab A, Altunbulakli C, Azkur K, Costa RA, Crameri R, et al. Interleukins (From IL-1 to IL-38), Interferons, Transforming Growth Factor Beta, and TNF-alpha: Receptors, Functions, and Roles in Diseases. J Allergy Clin Immunol (2016) 138(4):984-1010. doi: 10.1016/j.jaci.2016.06.033

79. Miller MC, Mayo KH. Chemokines From a Structural Perspective. Int J Mol Sci (2017) 18(10):2088. doi: 10.3390/ijms18102088

80. Oppenheim JJ, Matsushima K, Yoshimura T, Leonard EJ. The Activities of Cytokines are Pleiotropic and Interdependent. Immunol Lett (1987) 16(34):179-83. doi: 10.1016/0165-2478(87)90145-3

81. Ozaki K, Leonard WJ. Cytokine and Cytokine Receptor Pleiotropy and Redundancy. J Biol Chem (2002) 277(33):29355-8. doi: 10.1074/ jbc.R200003200

82. Aikawa N. Cytokine Storm in the Pathogenesis of Multiple Organ Dysfunction Syndrome Associated With Surgical Insults. Nihon Geka Gakkai zasshi (1996) 97(9):771-7.

83. Bown MJ, Nicholson ML, Bell PR, Sayers RD. Cytokines and Inflammatory Pathways in the Pathogenesis of Multiple Organ Failure Following Abdominal Aortic Aneurysm Repair. Eur J Vasc endovascular Surg Off $J$ Eur Soc Vasc Surg (2001) 22(6):485-95. doi: 10.1053/ejvs.2001.1522

84. De Gaudio AR, Romagnoli S. Sepsis and Septic Shock, Eighth ed. China: Elsevier Health Sciences (2019). pp. 836-48.

85. Lipnik-Stangelj M. Mediators of Inflammation as Targets for Chronic Pain Treatment. Mediators Inflammation (2013) 2013:783235-. doi: 10.1155/ 2013/783235

86. Zhang J-M, An J. Cytokines, Inflammation, and Pain. Int Anesthesiol Clin (2007) 45(2):27-37. doi: 10.1097/AIA.0b013e318034194e

87. Chen L, Deng H, Cui H, Fang J, Zuo Z, Deng J, et al. Inflammatory Responses and Inflammation-Associated Diseases in Organs. Oncotarget (2017) 9(6):7204-18. doi: 10.18632/oncotarget.23208

88. Robinson SM, Rasch S, Beer S, Valantiene I, Mickevicius A, Schlaipfer E, et al. Systemic Inflammation Contributes to Impairment of Quality of Life in Chronic Pancreatitis. Sci Rep (2019) 9(1):7318. doi: 10.1038/s41598-01943846-8

89. Henden AS, Hill GR. Cytokines in Graft-Versus-Host Disease. J Immunol (2015) 194(10):4604. doi: 10.4049/jimmunol.1500117

90. Tisoncik JR, Korth MJ, Simmons CP, Farrar J, Martin TR, Katze MG. Into the Eye of the Cytokine Storm. Microbiol Mol Biol Rev (2012) 76(1):16-32. doi: 10.1128/MMBR.05015-11

91. White M. Mediators of Inflammation and the Inflammatory Process. J Allergy Clin Immunol (1999) 103(3):S378-S81. doi: 10.1016/S0091-6749 (99)70215-0

92. Stone SF, Isbister GK, Shahmy S, Mohamed F, Abeysinghe C, Karunathilake $\mathrm{H}$, et al. Immune Response to Snake Envenoming and Treatment With Antivenom; Complement Activation, Cytokine Production and Mast Cell Degranulation. PloS Neglected Trop Dis (2013) 7(7):e2326. doi: 10.1371/ journal.pntd.0002326

93. Ibiapina HNS, Costa AG, Sachett JAG, Silva IM, Tarrago AM, Neves JCF, et al. An Immunological Stairway to Severe Tissue Complication Assembly in Bothrops Atrox Snakebites. Front Immunol (2019) 10:1882. doi: 10.3389/ fimmu.2019.01882

94. Markland FS. Snake Venoms and the Hemostatic System. Toxicon Off $J$ Int Soc Toxinol (1998) 36(12):1749-800. doi: 10.1016/S0041-0101(98) 00126-3

95. Luchini LSG, Pidde G, Squaiella-Baptistão CC, Tambourgi DV. Complement System Inhibition Modulates the Pro-Inflammatory Effects of a Snake Venom Metalloproteinase. Front Immunol (2019) 10:1137-. doi: 10.3389/fimmu.2019.01539

96. Rider P, Carmi Y, Cohen I. Biologics for Targeting Inflammatory Cytokines, Clinical Uses, and Limitations. Int J Cell Biol (2016) 2016:9259646-. doi: $10.1155 / 2016 / 9259646$ 
97. Zoccal KF, Gardinassi LG, Bordon KCF, Arantes EC, Marleau S, Ong H, et al. EP80317 Restrains Inflammation and Mortality Caused by Scorpion Envenomation in Mice. Front Pharmacol (2019) 10:171. doi: 10.3389/ fphar.2019.00171

98. Dalla-Favera R, Klein U. Germinal Centres: Role in B-cell Physiology and Malignancy. Nat Rev Immunol (2008) 8(1):22-33. doi: 10.1038/nri2217

99. Koretzky GA. Multiple Roles of CD4 and CD8 in T Cell Activation. J Immunol (2010) 185(5):2643-4. doi: 10.4049/jimmunol.1090076

100. Parker DC. T Cell-Dependent B Cell Activation. Annu Rev Immunol (1993) 11(1):331-60. doi: 10.1146/annurev.iy.11.040193.001555

101. Harwood NE, Batista FD. Early Events in B Cell Activation. Annu Rev Immunol (2010) 28(1):185-210. doi: 10.1146/annurev-immunol-030409101216

102. Restano-Cassulini R, Garcia W, Paniagua-Solís J, Possani L. Annex 5: Guidelines for the Production, Control and Regulation of Snake Antivenom Immunoglobulins: Replacement of Annex 2 of WHO Technical Report Series, No. 964. World Health Organization (2016). Report No.: 0512-3054 Contract No.: Report.

103. Fox S, Rathuwithana AC, Kasturiratne A, Lalloo DG, de Silva HJ. Underestimation of Snakebite Mortality by Hospital Statistics in the Monaragala District of Sri Lanka. Trans R Soc Trop Med Hygiene (2006) 100(7):693-5. doi: 10.1016/j.trstmh.2005.09.003

104. Gilliam LL, Carmichael RC, Holbrook TC, Taylor JM, Ownby CL, McFarlane D, et al. Antibody Responses to Natural Rattlesnake Envenomation and a Rattlesnake Toxoid Vaccine in Horses. Clin Vaccine Immunol (2013) 20 (5):732-7. doi: 10.1128/CVI.00004-13

105. Casadevall A. Passive Antibody Administration (Immediate Immunity) as a Specific Defense Against Biological Weapons. Emerg Infect Dis (2002) 8 (8):833-41. doi: 10.3201/eid0808.010516

106. Restano-Cassulini R, Garcia W, Paniagua-Solis JF, Possani LD. Antivenom Evaluation by Electrophysiological Analysis. Toxins (2017) 9(3):74. doi: 10.3390/toxins 9030074

107. Elgueta R, Benson MJ, de Vries VC, Wasiuk A, Guo Y, Noelle RJ. Molecular Mechanism and Function of CD40/CD40L Engagement in the Immune System. Immunol Rev (2009) 229(1):152-72. doi: 10.1111/j.1600065X.2009.00782.x

108. Magee CN, Boenisch O, Najafian N. The Role of Costimulatory Molecules in Directing the Functional Differentiation of Alloreactive T Helper Cells. Am J Transplant (2012) 12(10):2588-600. doi: 10.1111/j.1600-6143.2012. 04180.x

109. Vazquez MI, Catalan-Dibene J, Zlotnik A. B Cells Responses and Cytokine Production are Regulated by Their Immune Microenvironment. Cytokine (2015) 74(2):318-26. doi: 10.1016/j.cyto.2015.02.007

110. Shlomchik MJ, Weisel F. Germinal Center Selection and the Development of Memory B and Plasma Cells. Immunol Rev (2012) 247(1):52-63. doi: 10.1111/j.1600-065X.2012.01124.x

111. Khan U, Ghazanfar H. T Lymphocytes and Autoimmunity. Int Rev Cell Mol Biol (2018) 341:125-68. doi: 10.1016/bs.ircmb.2018.05.008

112. Rangel-Santos A, Lima C, Lopes-Ferreira M, Cardoso DF. Immunosuppresive Role of Principal Toxin (Crotoxin) of Crotalus Durissus Terrificus Venom. Toxicon Off J Int Soc Toxinol (2004) 44 (6):609-16. doi: 10.1016/j.toxicon.2004.07.004

113. Beeton C, Pennington MW, Wulff H, Singh S, Nugent D, Crossley G, et al. Targeting Effector Memory T Cells With a Selective Peptide Inhibitor of Kv1.3 Channels for Therapy of Autoimmune Diseases. Mol Pharmacol (2005) 67(4):1369-81. doi: 10.1124/mol.104.008193

114. Bencherif M, Lippiello PM, Lucas R, Marrero MB. Alpha7 Nicotinic Receptors as Novel Therapeutic Targets for Inflammation-Based Diseases. Cell Mol Life Sci (2011) 68(6):931-49. doi: 10.1007/s00018010-0525-1

115. Bagdány M, Batista CVF, Valdez-Cruz NA, Somodi S, Rodriguez De La Vega $\mathrm{RC}$, Licea AF, et al. Anuroctoxin, a New Scorpion Toxin of the $\alpha$-KTx 6 Subfamily, is Highly Selective for Kv1.3 Over IKCa1 Ion Channels of Human T Lymphocytes. Mol Pharmacol (2005) 67(4):1034-44. doi: 10.1124/ mol.104.007187

116. Waheed H, Moin SF, Choudhary MI. Snake Venom: From Deadly Toxins to Life-Saving Therapeutics. Curr Med Chem (2017) 24(17):1874-91. doi: $10.2174 / 09298673246666170605091546$
117. Pennington MW, Czerwinski A, Norton RS. Peptide Therapeutics From Venom: Current Status and Potential. Bioorg Med Chem (2018) 26(10):273858. doi: 10.1016/j.bmc.2017.09.029

118. Bogin O. Venom Peptides and Their Mimetics as Potential Drugs. Modulator (2005) 19:14-20.

119. Pineda SS, Undheim EA, Rupasinghe DB, Ikonomopoulou MP, King GF. Spider Venomics: Implications for Drug Discovery. Fut Med Chem (2014) 6 (15):1699-714. doi: 10.4155/fmc.14.103

120. King GF. Venoms as a Platform for Human Drugs: Translating Toxins Into Therapeutics. Expert Opin Biol Ther (2011) 11(11):1469-84. doi: 10.1517/ 14712598.2011.621940

121. Ryan RYM, Lutzky VP, Herzig V, Smallwood TB, Potriquet J, Wong Y, et al. Venom of the Red-Bellied Black Snake Pseudechis Porphyriacus Shows Immunosuppressive Potential. Toxins (2020) 12(11):674. doi: 10.3390/ toxins 12110674

122. Welton RE, Williams DJ, Liew D. Injury Trends From Envenoming in Australia, 2000-2013. Internal Med J (2017) 47(2):170-6. doi: 10.1111/ imj.13297

123. Mullins RJ, Wainstein BK, Barnes EH, Liew WK, Campbell DE. Increases in Anaphylaxis Fatalities in Australia From 1997 to 2013. Clin Exp Allergy (2016) 46(8):1099-110. doi: 10.1111/cea.12748

124. Pesek RD, Lockey RF. Management of Insect Sting Hypersensitivity: An Update. Allergy Asthma Immunol Res (2013) 5(3):129-37. doi: 10.4168/ aair.2013.5.3.129

125. Bilò MB. Anaphylaxis Caused by Hymenoptera Stings: From Epidemiology to Treatment. Allergy (2011) 66(95):35-7. doi: 10.1111/j.13989995.2011.02630.x

126. Curotto de Lafaille MA, Lafaille JJ, Graça L. Mechanisms of Tolerance and Allergic Sensitization in the Airways and the Lungs. Curr Opin Immunol (2010) 22(5):616-22. doi: 10.1016/j.coi.2010.08.014

127. Ollert M, Blank S. Anaphylaxis to Insect Venom Allergens: Role of Molecular Diagnostics. Curr Allergy Asthma Rep (2015) 15(5):26-. doi: 10.1007/s11882015-0527-z

128. Janeway CNational Library of M. Immunobiology: The Immune System in Health and Disease. Fifth ed. New York: Garland Pub (2001).

129. van Ree R, Hummelshøj L, Plantinga M, Poulsen LK, Swindle E. Allergic Sensitization: Host-immune Factors. Clin Trans Allergy (2014) 4(1):12. doi: 10.1186/2045-7022-4-12

130. Ellenbogen Y, Jiménez-Saiz R, Spill P, Chu DK, Waserman S, Jordana M. The Initiation of Th2 Immunity Towards Food Allergens. Int J Mol Sci (2018) 19 (5):1447. doi: 10.3390/ijms19051447

131. Thangam EB, Jemima EA, Singh H, Baig MS, Khan M, Mathias CB, et al. The Role of Histamine and Histamine Receptors in Mast Cell-Mediated Allergy and Inflammation: The Hunt for New Therapeutic Targets. Front Immunol (2018) 9(1873). doi: 10.3389/fimmu.2018.01873

132. Stitt JMD, Katial RMD. Venom Allergy. J Allergy Clin Immunol: In Pract (2015) 4(1):184-5. doi: 10.1016/j.jaip.2015.09.016

133. Lieberman P. Biphasic Anaphylactic Reactions. Ann allergy Asthma Immunol Off Publ Am Coll Allergy Asthma Immunol (2005) 95(3):217-26; quiz 26, 58. doi: 10.1016/S1081-1206(10)61217-3

134. Liu M, Yokomizo T. The Role of Leukotrienes in Allergic Diseases. Allergology Int (2015) 64(1):17-26. doi: 10.1016/j.alit.2014.09.001

135. Niedoszytko M, Bonadonna P, Oude Elberink JN, Golden DB. Epidemiology, Diagnosis, and Treatment of Hymenoptera Venom Allergy in Mastocytosis Patients. Immunol Allergy Clinics North America (2014) 34(2):365-81. doi: 10.1016/j.iac.2014.02.004

136. Philipp AMD, Ferdman RMMDM, Tam JSMD. Evaluation of Venom Allergy. Ann Allergy Asthma Immunol (2016) 117(4):344-7. doi: 10.1016/ j.anai.2016.08.012

137. Lieberman P. The Use of Antihistamines in the Prevention and Treatment of Anaphylaxis and Anaphylactoid Reactions. J Allergy Clin Immunol (1990) 86 (4 Pt 2):684-6. doi: 10.1016/S0091-6749(05)80241-6

138. Remigante A, Costa R, Morabito R, La Spada G, Marino A, Dossena S. Impact of Scyphozoan Venoms on Human Health and Current First Aid Options for Stings. Toxins (2018) 10(4):133. doi: 10.3390/toxins 10040133

139. Schiener M, Graessel A, Ollert M, Schmidt-Weber CB, Blank S. AllergenSpecific Immunotherapy of Hymenoptera Venom Allergy - Also a Matter of 
Diagnosis. Hum Vaccin Immunother (2017) 13(10):2467-81. doi: 10.1080/ 21645515.2017.1334745

140. Mattila P, Joenväärä S, Renkonen J, Toppila-Salmi S, Renkonen R. Allergy as an Epithelial Barrier Disease. Clin Trans Allergy (2011) 1(1):1-8. doi: 10.1186/2045-7022-1-5

141. Cegolon L, Heymann WC, Lange JH, Mastrangelo G. Jellyfish Stings and Their Management: A Review. Mar Drugs (2013) 11(2):523-50. doi: 10.3390/ md11020523

142. Montgomery L, Seys J, Mees J. To Pee, or Not to Pee: A Review on Envenomation and Treatment in European Jellyfish Species. Mar Drugs (2016) 14(7):127. doi: 10.3390/md14070127

143. Glatstein M, Adir D, Galil B, Scolnik D, Rimon A, Pivko-Levy D, et al. Pediatric Jellyfish Envenomation in the Mediterranean Sea. Eur J Emergency Med Off J Eur Soc Emergency Med (2018) 25(6):434-9. doi: 10.1097/ MEJ.0000000000000479

144. Horiike T, Nagai H, Kitani S. Identification of Allergens in the Box Jellyfish Chironex Yamaguchii That Cause Sting Dermatitis. Int Arch Allergy Immunol (2015) 167(2):73-82. doi: 10.1159/000434721

145. Friedel N, Scolnik D, Adir D, Glatstein M. Severe Anaphylactic Reaction to Mediterranean Jellyfish (Ropilhema Nomadica) Envenomation: Case Report. Toxicol Rep (2016) 3:427-9. doi: 10.1016/j.toxrep.2016.03.006

146. Uri S, Marina G, Liubov G. Severe Delayed Cutaneous Reaction Due to Mediterranean Jellyfish (Rhopilema Nomadica) Envenomation. Contact Dermatitis (2005) 52(5):282-3. doi: 10.1111/j.0105-1873.2005.00582.x

147. Kubota S, Nozawa A, Yanai T, Ozasa K, Mori S, Kurihara K. The Case of a 14-Year-Old Boy Who Experienced Anaphylaxis Due to Jellyfish (Mastigias Papua) Ingestion. Arerugi $=$ [Allergy] (2017) 66(6):809-12. doi: 10.15036/ arerugi.66.809

148. Cañas JA, Rodrigo-Muñoz JM, Rondon-Cepeda SH, Bordehore C, Fernández-Nieto M, del Pozo V. Jellyfish Collagen: A New Allergen in the Beach. Ann Allergy Asthma Immunol (2018) 120(4):430-1. doi: 10.1016/ j.anai.2018.01.018

149. Tibballs J, Yanagihara AA, Turner HC, Winkel K. Immunological and Toxinological Responses to Jellyfish Stings. Inflammation Allergy Drug Targets (2011) 10(5):438-46. doi: 10.2174/187152811797200650

150. Buttaravoli PMDF, Leffler SMMDF. Marine Envenomations, 3rd ed. London: Elsevier Health Sciences (2012). pp. 574-80.

151. Morabito R, Marino A, La Spada G. Nematocytes' Activation in Pelagia Noctiluca (Cnidaria, Scyphozoa) Oral Arms. J Comp Physiol A (2012) 198 (6):419-26. doi: 10.1007/s00359-012-0720-7

152. Varney SMMDFF. Bites and Stings, Sixth ed. Philaldelphia: Elsevier (2016). pp. 474-83.e1

153. Badré S. Bioactive Toxins From Stinging Jellyfish. Toxicon Off $J$ Int Soc Toxinol (2014) 91:114-25. doi: 10.1016/j.toxicon.2014.09.010

154. Nagai H, Takuwa K, Nakao M, Ito E, Miyake M, Noda M, et al. Novel Proteinaceous Toxins From the Box Jellyfish (Sea Wasp) Carybdea Rastoni. Biochem Biophys Res Commun (2000) 275(2):582-8. doi: 10.1006/ bbrc.2000.3353

155. Nagai H, Takuwa K, Nakao M, Sakamoto B, Crow GL, Nakajima T. Isolation and Characterization of a Novel Protein Toxin From the Hawaiian Box Jellyfish (Sea Wasp) Carybdea Alata. Biochem Biophys Res Commun (2000) 275(2):589-94. doi: 10.1006/bbrc.2000.3352

156. Nagai H, Takuwa-Kuroda K, Nakao M, Oshiro N, Iwanaga S, Nakajima T. A Novel Protein Toxin From the Deadly Box Jellyfish (Sea Wasp, HabuKurage) Chiropsalmus Quadrigatus. Biosci Biotechnol Biochem (2002) 66 (1):97-102. doi: 10.1271/bbb.66.97

157. Brinkman DL, Konstantakopoulos N, McInerney BV, Mulvenna J, Seymour JE, Isbister GK, et al. Chironex Fleckeri (Box Jellyfish) Venom Proteins: Expansion of a Cnidarian Toxin Family That Elicits Variable Cytolytic and Cardiovascular Effects. J Biol Chem (2014) 289(8):4798-812. doi: 10.1074/ jbc.M113.534149

158. Kramp PL. Synopsis of the Medusae of the World. J Marine Biol Assoc U Kingdom (1961) 40:7-382. doi: 10.1017/S0025315400007347

159. Tibballs J. Australian Venomous Jellyfish, Envenomation Syndromes, Toxins and Therapy. Toxicon Off J Int Soc Toxinol (2006) 48(7):830-59. doi: 10.1016/j.toxicon.2006.07.020

160. White J. Venom. In: J Payne-James and RW Byard, editors. Encyclopedia of Forensic and Legal Medicine, 2nd ed. Oxford: Elsevier (2016). p. 622-38.
161. Fox JW. Venoms and Poisons From Marine Organisms. In: L Goldman and AI Schafer, editors. Goldman's Cecil Medicine, 4th ed. Philadelphia: W.B. Saunders (2012). p. 697-700.

162. Brinchmann BC, Bayat M, Brøgger T, Muttuvelu DV, Tjønneland A, Sigsgaard T. A Possible Role of Chitin in the Pathogenesis of Asthma and Allergy. Ann Agric Environ Med (2011) 18(1):7-12.

163. Burton OT, Zaccone P. The Potential Role of Chitin in Allergic Reactions. Trends Immunol (2007) 28(10):419-22. doi: 10.1016/j.it.2007.08.005

164. Lee CG, Da Silva CA, Dela Cruz CS, Ahangari F, Ma B, Kang M-J, et al. Role of Chitin and Chitinase/Chitinase-Like Proteins in Inflammation, Tissue Remodeling, and Injury. Annu Rev Physiol (2011) 73:479-501. doi: 10.1146/ annurev-physiol-012110-142250

165. Inomata N, Chin K, Aihara M. Anaphylaxis Caused by Ingesting Jellyfish in a Subject With Fermented Soybean Allergy: Possibility of Epicutaneous Sensitization to Poly-Gamma-Glutamic Acid by Jellyfish Stings. J Dermatol (2014) 41(8):752-3. doi: 10.1111/1346-8138.12542

166. Inomata N, Miyakawa M, Aihara M. Surfing as a Risk Factor for Sensitization to Poly( $\gamma$-Glutamic Acid) in Fermented Soybeans, Natto, Allergy. Allergology Int (2018) 67(3):341-6. doi: 10.1016/j.alit. 2017.11.001

167. Shimabukuro-Vornhagen A, Gödel P, Subklewe M, Stemmler HJ, Schlößer HA, Schlaak M, et al. Cytokine Release Syndrome. J Immunother Cancer (2018) 6(1):56. doi: 10.1186/s40425-018-0343-9

168. Suntharalingam G, Perry MR, Ward S, Brett SJ, Castello-Cortes A, Brunner $\mathrm{MD}$, et al. Cytokine Storm in a Phase 1 Trial of the anti-CD28 Monoclonal Antibody TGN1412. New Engl J Med (2006) 355(10):1018-28. doi: 10.1056/ NEJMoa063842

169. Wood AJ, Darbyshire J. Injury to Research Volunteers; the Clinical-Research Nightmare. New Engl J Med (2006) 354(18):1869-71. doi: 10.1056/ NEJMp068082

170. Reis MB, Rodrigues FL, Lautherbach N, Kanashiro A, Sorgi CA, Alyne FGM, et al. Interleukin-1 Receptor-Induced PGE2 Production Controls Acetylcholine-Mediated Cardiac Dysfunction and Mortality During Scorpion Envenomation. Nat Commun (2020) 11(1):5433-. doi: 10.1038/ s41467-020-19232-8

171. Tiong K. Irukandji Syndrome, Catecholamines, and Mid-Ventricular Stress Cardiomyopathy. Eur Heart J - Cardiovasc Imaging (2008) 10(2):334-6. doi: 10.1093/ejechocard/jen246

172. Petricevich VL. Scorpion Venom and the Inflammatory Response. Mediators Inflammation (2010) 2010:903295-. doi: 10.1155/2010/903295

173. Santos MS, Silva CG, Neto BS, Grangeiro Junior CR, Lopes VH, Teixeira Junior AG, et al. Clinical and Epidemiological Aspects of Scorpionism in the World: A Systematic Review. Wilderness Environ Med (2016) 27(4):504-18. doi: 10.1016/j.wem.2016.08.003

174. Comellas AP, Pesce LM, Azzam Z, Saldias FJ, Sznajder JI. Scorpion Venom Decreases Lung Liquid Clearance in Rats. Am J Respir Crit Care Med (2003) 167(8):1064-7. doi: 10.1164/rccm.200207-688OC

175. Pessini AC, de Souza AM, Faccioli LH, Gregório ZMO, Arantes EC. Time Course of Acute-Phase Response Induced by Tityus Serrulatus Venom and TsTX-I in Mice. Int Immunopharmacol (2003) 3(5):765-74. doi: 10.1016/ S1567-5769(03)00078-X

176. Santhosh KN, Pavana D, Thippeswamy NB. Impact of Scorpion venoWm as an Acute Stressor on the Neuroendocrine-Immunological Network. Toxicon Off J Int Soc Toxinol (2016) 122:113-8. doi: 10.1016/ j.toxicon.2016.09.021

177. Megdad-Lamraoui A, Adi-Bessalem S, Laraba-Djebari F. Cerebrospinal Inflammatory Response Following Scorpion Envenomation: Role of Histamine H1 and H3 Receptors. Inflammopharmacology (2019) 27 (3):589-601. doi: 10.1007/s10787-018-00553-6

178. Zoccal KF, da SilvaBitencourt C, CA S. (2013) 61(1):1-10.

179. Muraoka N, Nara K, Tamura F, Kojima H, Yamakawa H, Sadahiro T, et al. Role of cyclooxygenase-2-mediated Prostaglandin E2-Prostaglandin E Receptor 4 Signaling in Cardiac Reprogramming. Nat Commun (2019) 10 (1):674. doi: 10.1038/s41467-019-08626-y

180. Zoccal KF, Gardinassi LG, AtérioSorgi C, Meirelles AFG, Bordon KCF, Glezer I, et al. CD36 Shunts Eicosanoid Metabolism to Repress CD14 Licensed interleukin-1beta Release and Inflammation. Front Immunol (2018) 9:890. doi: 10.3389/fimmu.2018.00890 
181. Mégarbane B, Abroug F, Soulaymani R, Comellas AP, Pesce LM, Patil SN, et al. Scorpion Envenomation. New Engl J Med (2014) 371(16):1557-60. doi: 10.1056/NEJMc1410354

182. Board on Health Sciences P, Institute of M, Committee on Conflict of Interest in Medical Research E, Practice. The Pathway From Idea to Regulatory Approval: Examples for Drug Development. L Bernard and JF Marilyn, editors. Washington (DC): National Academies Press (2009). p. $375-83$.

183. Currie BJ, Jacups SP. Prospective Study of Chironex Fleckeri and Other Box Jellyfish Stings in the "Top End" of Australia's Northern Territory. Med J Aust (2005) 183(11-12):631-6. doi: 10.5694/j.1326-5377.2005.tb00062.x

184. Tibballs J, Li R, Tibballs HA, Gershwin LA, Winkel KD. Australian Carybdeid Jellyfish Causing "Irukandji Syndrome". Toxicon Off J Int Soc Toxinol (2012) 59(6):617-25. doi: 10.1016/j.toxicon.2012.01.006

185. Fenner P, Carney I. The Irukandji Syndrome. A Devastating Syndrome Caused by a North Australian Jellyfish. Australian Family Physician (1999) 28(11):1131-7.

186. Huynh TT, Pereira P, Mulcahy R, Cullen P, Seymour J, Carrette T, et al. Severity of Irukandji Syndrome and Nematocyst Identification From Skin Scrapings. Med J Aust (2003) 178(1):38-41. doi: 10.5694/j.13265377.2003.tb05041.x

187. Barnes JH. Cause and Effect in Irukandji Stingings. Med J Aust (1964) 1:897904. doi: $10.5694 / j .1326-5377.1964 . t b 114424 . x$

188. Flecker H. Irukandji Sting to North Queensland Bathers Without Production of Weals But With Severe General Symptoms. Med J Aust (1952) 2(3):89-91. doi: 10.5694/j.1326-5377.1952.tb100081.x

189. Courtney R, Browning S, Seymour J. Early Life History of the 'Irukandji' Jellyfish Carukia Barnesi. PloS One (2016) 11(3):e0151197-e. doi: 10.1371/ journal.pone. 0151197

190. Courtney R, Sachlikidis N, Jones R, Seymour J. Prey Capture Ecology of the Cubozoan Carukia Barnesi. PloS One (2015) 10(5):e0124256. doi: 10.1371/ journal.pone. 0124256

191. McIver LJMM, Tjhung IGMD, Parish STMDTM, Derkenne RCBADTM, Kippin ANMM. Irukandji Syndrome in the Torres Strait: A Series of 8 Cases. Wilderness Environ Med (2011) 22(4):338-42. doi: 10.1016/ j.wem.2011.08.002

192. Tibballs J. Envenomation. In: Bersten A, Handy J, editors. Oh's Intensive Care Manual. Eighth ed. China: Elsevier (2019). pp. 1006-18.e2.

193. Nickson CP, Waugh EB, Jacups SP, Currie BJ. Irukandji Syndrome Case Series From Australia's Tropical Northern Territory. Ann Emergency Med (2009) 54(3):395-403. doi: 10.1016/j.annemergmed.2009.03.022

194. Nickson CP, Currie BJ, Fenner PJ. Priapism and Irukandji Syndrome. Ann Emergency Med (2010) 55(6):581-2. doi: 10.1016/j.annemergmed. 2010.01.006

195. Little M, Pereira P, Mulcahy R, Cullen P, Carrette T, Seymour J. Severe Cardiac Failure Associated With Presumed Jellyfish. Sting Irukandji syndrome? Anaesthesia Intensive Care (2003) 31(6):642-7. doi: 10.1177/ $0310057 \mathrm{X} 0303100605$

196. Fenner PJ, Williamson JA, Gunawardane K, Murtha W, Burnett JW, Colquhoun DM, et al. The "Irukandji Syndrome" and Acute Pulmonary Oedema. Med J Aust (1988) 149(3):150-6. doi: 10.5694/j.13265377.1988.tb120544.x

197. Pereira P, Barry J, Corkeron M, Keir P, Little M, Seymour J. Intracerebral Hemorrhage and Death After Envenoming by the Jellyfish Carukia Barnesi. Clin Toxicol (Philadelphia Pa) (2010) 48(4):390-2. doi: 10.3109/ 15563651003662675

198. Ramasamy S, Isbister GK, Seymour JE, Hodgson WC. The In Vivo Cardiovascular Effects of the Irukandji Jellyfish (Carukia Barnesi) Nematocyst Venom and a Tentacle Extract in Rats. Toxicol Lett (2005) 155(1):135-41. doi: 10.1016/j.toxlet.2004.09.004

199. Bailey PM. Fatal Envenomation by Jellyfish Causing Irukandji Syndrome. Med J Aust (2003) 178(3):139; author reply -40. doi: 10.5694/j.13265377.2003.tb05108.x

200. Winkel KD, Tibballs J, Molenaar P, Lambert G, Coles P, Ross-Smith M, et al. Cardiovascular Actions of the Venom From the Irukandji (Carukia Barnesi) Jellyfish: Effects in Human, Rat and Guinea-Pig Tissues In Vitro and in Pigs In Vitro. Clin Exp Pharmacol Physiol (2005) 32(9):777-88. doi: 10.1111/ j.1440-1681.2005.04258.x
201. Corkeron M, Pereira P, Makrocanis C. Early Experience With Magnesium Administration in Irukandji Syndrome. Anaesthesia Intensive Care (2004) 32 (5):666-9. doi: 10.1177/0310057X0403200510

202. McCullagh N, Pereira P, Cullen P, Mulcahy R, Bonin R, Little M, et al. Randomised Trial of Magnesium in the Treatment of Irukandji Syndrome. Emergency Med Australasia EMA (2012) 24(5):560-5. doi: 10.1111/j.17426723.2012.01602.x

203. Rathbone J, Franklin R, Gibbs C, Williams D. Review Article: Role of Magnesium Sulphate in the Management of Irukandji Syndrome: A Systematic Review. Emergency Med Australasia EMA (2017) 29(1):9-17. doi: 10.1111/1742-6723.12694

204. Sugimoto J, Romani AM, Valentin-Torres AM, Luciano AA, Ramirez Kitchen $\mathrm{CM}$, Funderburg N, et al. Magnesium Decreases Inflammatory Cytokine Production: A Novel Innate Immunomodulatory Mechanism. J Immunol (Baltimore Md 1950) (2012) 188(12):6338-46. doi: 10.4049/jimmunol.1101765

205. Siddall E, Radhakrishnan J. Capillary Leak Syndrome: A Cytokine and Catecholamine Storm? Kidney Int (2019) 95(5):1009-11. doi: 10.1016/ j.kint.2019.03.001

206. Staedtke V, Bai RY, Kim K, Darvas M, Davila ML, Riggins GJ, et al. Disruption of a Self-Amplifying Catecholamine Loop Reduces Cytokine Release Syndrome. Nature (2018) 564(7735):273-7. doi: 10.1038/s41586018-0774-y

207. Hwang D-S, Kim SK, Bae H. Therapeutic Effects of Bee Venom on Immunological and Neurological Diseases. Toxins (2015) 7(7):2413-21. doi: $10.3390 /$ toxins 7072413

208. Grgic I, Wulff H, Eichler I, Flothmann C, Kohler R, Hoyer J. Blockade of Tlymphocyte Kca3.1 and Kv1.3 Channels as Novel Immunosuppression Strategy to Prevent Kidney Allograft Rejection. Transplant Proc (2009) 41 (6):2601-6. doi: 10.1016/j.transproceed.2009.06.025

209. Kou JQ, Han R, Xu YL, Ding XL, Wang SZ, Chen CX, et al. Differential Effects of Naja Naja Atra Venom on Immune Activity. Evidence-Based complementary Altern Med eCAM (2014) 2014:287631. doi: 10.1155/2014/287631

210. Nam S, Ko E, Park SK, Ko S, Jun CY, Shin MK, et al. Bee Venom Modulates Murine Th1/Th2 Lineage Development. Int Immunopharmacol (2005) 5 (9):1406-14. doi: 10.1016/j.intimp.2005.03.011

211. Xu YL, Kou JQ, Wang SZ, Chen CX, Qin ZH. Neurotoxin From Naja Naja Atra Venom Inhibits Skin Allograft Rejection in Rats. Int Immunopharmacol (2015) 28(1):188-98. doi: 10.1016/j.intimp.2015.05.040

212. Grafton G, Thwaite L. Calcium Channels in Lymphocytes. Immunology (2001) 104(2):119-26. doi: 10.1046/j.0019-2805.2001.01321.x

213. Cahalan MD, Wulff H, Chandy KG. Molecular Properties and Physiological Roles of Ion Channels in the Immune System. J Clin Immunol (2001) 21 (4):235-52. doi: 10.1023/A:1010958907271

214. Feske S, Wulff H, Skolnik EY. Ion Channels in Innate and Adaptive Immunity. Annu Rev Immunol (2015) 33:291-353. doi: 10.1146/annurevimmunol-032414-112212

215. Hu L, Wang T, Gocke AR, Nath A, Zhang H, Margolick JB, et al. Blockade of Kv1.3 Potassium Channels Inhibits Differentiation and Granzyme B Secretion of Human CD8+ T Effector Memory Lymphocytes. PloS One (2013) 8(1):e54267. doi: 10.1371/journal.pone.0054267

216. Schmalhofer WA, Bao J, McManus OB, Green B, Matyskiela M, Wunderler $D$, et al. Identification of a New Class of Inhibitors of the Voltage-Gated Potassium Channel, Kv1.3, With Immunosuppressant Properties. Biochemistry (2002) 41(24):7781-94. doi: 10.1021/bi025722c

217. Wulff H, Calabresi PA, Allie R, Yun S, Pennington M, Beeton C, et al. The Voltage-Gated $\mathrm{Kv1} .3 \mathrm{~K}(+)$ Channel in Effector Memory T Cells as New Target for MS. J Clin Invest (2003) 111(11):1703-13. doi: 10.1172/JCI16921

218. Alama A, Bruzzo C, Cavalieri Z, Forlani A, Utkin Y, Casciano I, et al. Inhibition of the Nicotinic Acetylcholine Receptors by Cobra Venom $\alpha$ Neurotoxins: Is There a Perspective in Lung Cancer Treatment? PloS One (2011) 6(6):e20695-e. doi: 10.1371/journal.pone.0020695

219. Zhao Y, Huang J, Yuan X, Peng B, Liu W, Han S, et al. Toxins Targeting the Kv1.3 Channel: Potential Immunomodulators for Autoimmune Diseases. Toxins (2015) 7(5):1749-64. doi: 10.3390/toxins7051749

220. Koshy S, Huq R, Tanner MR, Atik MA, Porter PC, Khan FS, et al. Blocking KV1.3 Channels Inhibits Th2 Lymphocyte Function and Treats a Rat Model of Asthma. J Biol Chem (2014) 289(18):12623-32. doi: 10.1074/ jbc.M113.517037 
221. Matheu MP, Beeton C, Garcia A, Chi V, Rangaraju S, Safrina O, et al. Imaging of Effector Memory $\mathrm{T}$ Cells During a Delayed-Type Hypersensitivity Reaction and Suppression by Kv1.3 Channel Block. Immunity (2008) 29(4):602-14. doi: 10.1016/j.immuni.2008.07.015

222. Panyi G, Possani LD, Rodriguez de la Vega RC, Gaspar R, Varga Z. K+ Channel Blockers: Novel Tools to Inhibit $\mathrm{T}$ Cell Activation Leading to Specific Immunosuppression. Curr Pharm Design (2006) 12(18):2199-220. doi: $10.2174 / 138161206777585120$

223. Toldi G, Bajnok A, Dobi D, Kaposi A, Kovács L, Vásárhelyi B, et al. The Effects of Kv1.3 and IKCa1 Potassium Channel Inhibition on Calcium Influx of Human Peripheral T Lymphocytes in Rheumatoid Arthritis. Immunobiology (2012) 218(3):311-6.

224. Zhang S, Wang X, Ju C, Zhu L, Du Y, Gao C. Blockage of K(Ca)3.1 and Kv1.3 Channels of the B Lymphocyte Decreases the Inflammatory Monocyte Chemotaxis. Int Immunopharmacol (2016) 31:266-71.

225. Ali SA, Alam M, Abbasi A, Kalbacher H, Schaechinger TJ, Hu Y, et al. Structure-Activity Relationship of a Highly Selective Peptidyl Inhibitor of Kv1.3 Voltage-Gated K+-channel From Scorpion (B. Sindicus) Venom. Int J Pept Res Ther (2014) 20(1):19-32.

226. Cahalan MD, Chandy KG. The Functional Network of Ion Channels in T Lymphocytes. Immunol Rev (2009) 231(1):59-87.

227. Pennington MW, Beeton C, Galea CA, Smith BJ, Chi V, Monaghan KP, et al. Engineering a Stable and Selective Peptide Blocker of the Kv1.3 Channel in T Lymphocytes. Mol Pharmacol (2009) 75(4):762-73. doi: 10.1124/mol.108.052704

228. Mouhat S, Jouirou B, Mosbah A, De Waard M, Sabatier J-M. Diversity of Folds in Animal Toxins Acting on Ion Channels. Biochem J (2004) 378 (3):717-26. doi: 10.1042/bj20031860

229. Herzig V, King GF. The Cystine Knot is Responsible for the Exceptional Stability of the Insecticidal Spider Toxin $\omega$-Hexatoxin-Hvla. Toxins (2015) 7 (10):4366-80. doi: 10.3390/toxins7104366

230. Liu Y-L, Lin H-M, Zou R, Wu J-C, Han R, Raymond LN, et al. Suppression of Complete Freund's Adjuvant-Induced Adjuvant Arthritis by Cobratoxin. Acta Pharmacol Sin (2009) 30(2):219-27. doi: 10.1038/aps.2008.20

231. Choi MS, Choi T, Park S, Lee G, Haam K-K, Hong M-C, et al. Bee Venom Ameliorates Ovalbumin Induced Allergic Asthma Via Modulating CD4 +CD25+ Regulatory T Cells in Mice. Cytokine (2013) 61(1):256-65. doi: 10.1016/j.cyto.2012.10.005
232. Elias JA, Lee CG, Zheng T, Ma B, Homer RJ, Zhu Z. New Insights Into the Pathogenesis of Asthma. J Clin Invest (2003) 111(3):291-7. doi: 10.1172/JCI17748

233. Gilmore TD. Introduction to NF-kappaB: Players, Pathways, Perspectives. Oncogene (2006) 25(51):6680. doi: 10.1038/sj.onc.1209954

234. Gu SM, Park MH, Hwang CJ, Song HS, Lee US, Han SB, et al. Bee Venom Ameliorates Lipopolysaccharide-Induced Memory Loss by Preventing NFkappaB Pathway. J Neuroinflamm (2015) 12(1):124. doi: 10.1186/s12974015-0344-2

235. Lawrence T. The Nuclear Factor NF-Kappa B Pathway in Inflammation. Cold Spring Harbor Perspect Biol (2009) 1(6):a001651. doi: 10.1101/ cshperspect.a001651

236. Palm Noah W, Rosenstein Rachel K, Yu S, Schenten Dominik D, Florsheim E, Medzhitov R. Bee Venom Phospholipase A2 Induces a Primary Type 2 Response That is Dependent on the Receptor ST2 and Confers Protective Immunity. Immunity (2013) 39(5):976-85. doi: 10.1016/j.immuni. 2013.10.006

237. Garg R, Juncadella IJ, Ramamoorthi N, Ashish, Ananthanarayanan SK, Thomas V, et al. Cutting Edge: CD4 is the Receptor for the Tick Saliva Immunosuppressor, Salp15. J Immunol (2006) 177(10):6579-83. doi: 10.4049/jimmunol.177.10.6579

238. Zhang M, Caragine T, Wang H, Cohen PS, Botchkina G, Soda K, et al. Spermine Inhibits Proinflammatory Cytokine Synthesis in Human Mononuclear Cells: A Counterregulatory Mechanism That Restrains the Immune Response. J Exp Med (1997) 185(10):1759-68. doi: 10.1084/ jem.185.10.1759

Conflict of Interest: The authors declare that the research was conducted in the absence of any commercial or financial relationships that could be construed as a potential conflict of interest.

Copyright (c) 2021 Ryan, Seymour, Loukas, Lopez, Ikonomopoulou and Miles. This is an open-access article distributed under the terms of the Creative Commons Attribution License (CC BY). The use, distribution or reproduction in other forums is permitted, provided the original author(s) and the copyright owner(s) are credited and that the original publication in this journal is cited, in accordance with accepted academic practice. No use, distribution or reproduction is permitted which does not comply with these terms. 